\title{
Mutual dependence of the MRTF-SRF and YAP-TEAD pathways in cancer-associated fibroblasts is indirect and mediated by cytoskeletal dynamics
}

\author{
Charles T. Foster, Francesco Gualdrini, ${ }^{1}$ and Richard Treisman \\ Signalling and Transcription Group, Francis Crick Institute, London NW1 1AT, United Kingdom
}

Both the MRTF-SRF and the YAP-TEAD transcriptional regulatory networks respond to extracellular signals and mechanical stimuli. We show that the MRTF-SRF pathway is activated in cancer-associated fibroblasts (CAFs). The MRTFs are required in addition to the YAP pathway for CAF contractile and proinvasive properties. We compared MRTF-SRF and YAP-TEAD target gene sets and identified genes directly regulated by one pathway, the other, or both. Nevertheless, the two pathways exhibit mutual dependence. In CAFs, expression of direct MRTF-SRF genomic targets is also dependent on YAP-TEAD activity, and, conversely, YAP-TEAD target gene expression is also dependent on MRTF-SRF signaling. In normal fibroblasts, expression of activated MRTF derivatives activates YAP, while activated YAP derivatives activate MRTF. Cross-talk between the pathways requires recruitment of MRTF and YAP to DNA via their respective DNA-binding partners (SRF and TEAD) and is therefore indirect, arising as a consequence of activation of their target genes. In both CAFs and normal fibroblasts, we found that YAP-TEAD activity is sensitive to MRTF-SRF-induced contractility, while MRTF-SRF signaling responds to YAP-TEAD-dependent TGF $\beta$ signaling. Thus, the MRF-SRF and YAP-TEAD pathways interact indirectly through their ability to control cytoskeletal dynamics.

[Keywords: transcription; MRTF; SRF; YAP; TEAD; mechanotransduction; cancer-associated fibroblast]

Supplemental material is available for this article.

Received July 10, 2017; revised version accepted December 12, 2017.

The interface between mechanical force and gene expression is central to our understanding of normal and transformed cell behavior (Schwartz 2010; DuFort et al. 2011). Variation in extracellular matrix (ECM) stiffness and cell adhesion can affect gene expression programs to promote alternative cell fate choices (Engler et al. 2006; Discher et al. 2009), while, in cancer, malignant progression of solid tumors is associated with increased stiffening of the tumor microenvironment (Paszek et al. 2005; Levental et al. 2009; Schedin and Keely 2011). Cancer-associated fibroblasts (CAFs) play an important role in cancer progression. These myofibroblast-like cells, which express high levels of aSMA, promote cancer cell growth, invasion, and metastasis by paracrine signaling and ECM remodeling (for review, see Kalluri 2016).

The MRTF and YAP transcriptional pathways contribute to the response to mechanical stress. The MRTFs-

\footnotetext{
${ }^{1}$ Present address: Natoli Group, FIRC (Italian Foundation for Cancer Research) Institute of Molecular Oncology Foundation (IFOM)-European Institute of Oncology (IEO) Campus, 20139 Milan, Italy Corresponding author: richard.treisman@crick.ac.uk

Article published online ahead of print. Article and publication date are online at http://www.genesdev.org/cgi/doi/10.1101/gad.304501.117. Freely available online through the Genes \& Development Open Access option.
}

which are recruited to DNA by their partner, SRF-control expression of dozens of cytoskeletal genes, including aSMA (for review, see Olson and Nordheim 2010). They respond to Rho-GTPase signals, directly sensing changes in G-actin concentration via their regulatory RPEL domain, and accumulate in the nucleus when G-actin levels are low (Cen et al. 2003; Miralles et al. 2003; Vartiainen et al. 2007). The MRTFs can also be activated by substrate stiffness or direct integrin engagement (Zhao et al. 2007; Buxboim et al. 2014; Esnault et al. 2014). YAP and TAZ, which bind DNA in association with members of the TEAD family of DNA-binding cofactors, were first characterized as effectors of the Hippo growth control pathway (for review, see Meng et al. 2016). In addition, they also respond to Rho-GTPase signaling, accumulating in the nucleus in response to high cytoskeletal tension induced by mechanical cues (Dupont et al. 2011; Wada et al. 2011; Das et al. 2016). Previous studies have shown that YAP is activated in CAFs and that YAP-TEAD signaling maintains their contractile and proinvasive properties (Calvo et al. 2013; Dupont 2016).

(C) 2018 Foster et al. This article, published in Genes \& Development, is available under a Creative Commons License (Attribution 4.0 International), as described at http://creativecommons.org/licenses/by/4.0/. 
Several observations suggest that the MRTFs and YAP/ TAZ may functionally interact even though they do not share a common DNA targeting factor. The multiplicity of cytoskeletal genes and components of the YAP-TAZ interactome among direct MRTF-SRF genomic targets suggests the possibility of indirect pathway cross-talk (Dupont et al. 2011; Esnault et al. 2014). Some genes, such as Ctgf, Cyr61, and Ankrd1, contain binding sites for both SRF and TEAD and thus represent shared targets (for example, see Latinkic et al. 1991; Zhang et al. 2011). Moreover, YAP/TAZ and the MRTFs can physically interact, although it remains unclear whether this allows their recruitment to DNA independently of their own DNAbinding partners (Speight et al. 2016; Yu et al. 2016; Kim et al. 2017).

Here we investigate the relationship between MRTFSRF and YAP-TEAD signaling in CAFs and normal fibroblasts. We show that CAFs exhibit elevated MRTF activity, which is required for their contractile and proinvasive properties. Expression of YAP-TEAD genomic targets and expression of MRTF-SRF genomic targets are mutually dependent even when they are directly targeted by only one of the pathways. Finally, we show that activation of either pathway potentiates the activity of the other indirectly and that this depends on cytoskeletal dynamics.

\section{Results}

\section{MRTF-SRF signaling is activated in CAFs}

A previous analysis of stromal fibroblasts associated with tumors in the MMTV-PyMT mouse mammary carcinoma model suggested that expression of MRTF-SRF target genes may be increased in CAFs (Calvo et al. 2013). We compared that data set with our previous ChIP-seq (chromatin immunoprecipitation [ChIP] combined with highthroughput sequencing) analysis of MRTF-SRF target genes in serum-stimulated NIH3T3 fibroblasts (Esnault et al. 2014; Gualdrini et al. 2016). A substantial number of MRTF-SRF target genes, predominantly associated with cytoskeletal regulation, including the myofibroblast activation marker aSMA (Acta2), increased with severity of disease stage (Fig. 1A; Table 1A; Supplemental Table S1). The MRTF-SRF target gene data set also exhibited significant overlap with genes overexpressed in myofibroblastic CAFs associated with human pancreatic ductal adenocarcinoma (PDAC) (Table 1A; see the Discussion; Ohlund et al. 2017).

In fibroblasts, extracellular signaling through the Rhoactin pathway promotes MRTF nuclear accumulation (Miralles et al. 2003; Vartiainen et al. 2007). Although normal mammary fibroblasts (NFs) exhibited predominantly cytoplasmic and pancellular localization of MRTF-A in serum-starved conditions, the majority of CAF1 and CAF2 cells exhibited nuclear MRTF-A (Fig. 1B). Consistent with this, an MRTF-SRF reporter gene exhibited increased activity in CAFs compared with NFs (Fig. 1C) even though MRTF-A and MRTF-B expression was comparable in the two cell types (Supplemental Fig. S1A).
A

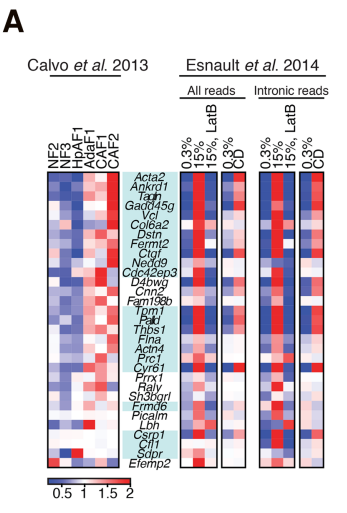

C
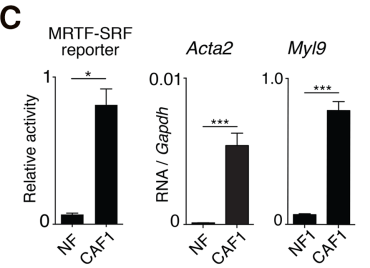

B

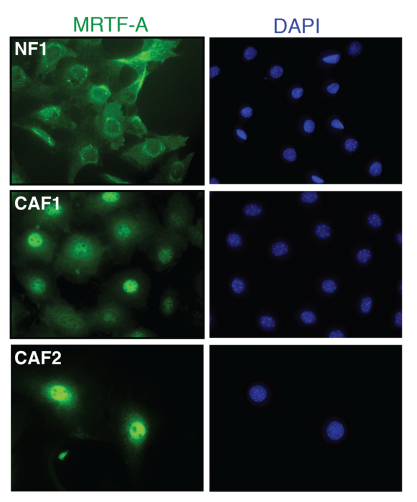

D
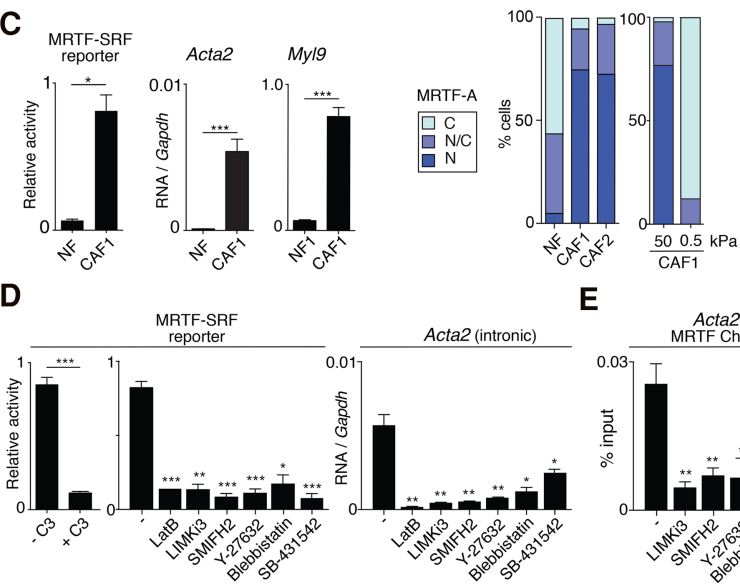

E
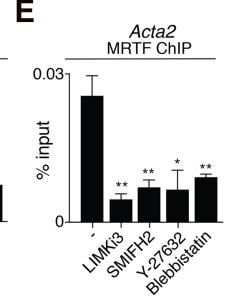

Figure 1. The MRTF-SRF pathway is activated in CAFs. $(A)$ Heat map expression profiles of MRTF-SRF target genes (Esnault et al. 2014) that are overexpressed in CAFs (Calvo et al. 2013). (Left) Fibroblasts from various stages of the mouse PyMT mammary tumor model (Calvo et al. 2013). (Right) NIH3T3 fibroblasts stimulated either with serum; with or without latrunculin B (LatB), which inhibits MRTF activation; or with cytochalasin D (CD), which specifically activates the MRTFs by competing for G-actin binding (Vartiainen et al. 2007). Genes shaded in blue are cytoskeletal components or regulators. $(B, t o p)$ Immunofluorescence microscopy of MRTF-A in normal mammary fibroblasts (NF1), CAF1, CAF2. (Bottom) MRTF-A localization in NFs and CAFs and in CAF1 cells plated on stiff and soft polyacrylamide hydrogels. (C) Higher cytoplasmic concentration; (N/C) equal concentration over the whole cell; $(\mathrm{N})$ higher nuclear concentration. (C) Comparison of MRTF-SRF reporter gene activity and Acta2 and Myl9 transcripts in NF1 and CAF1 cells. Data are mean \pm SEM. $n=3 .\left({ }^{*}\right) P<0.05$. (D) CAF1 cells were transfected with MRTF-SRF reporter and C3 transferase expression plasmids and treated with inhibitors as indicated before analysis of reporter activity or Acta2 transcripts. Data are mean \pm SEM. $n=3$. $\left(^{* * *}\right) P<$ 0.001 ; (**) $P<0.01$; (*) $P<0.05$ by $t$-test. $(E)$ ChIP analysis of MRTF-A in inhibitor-treated CAF1 cells. Data are mean \pm SEM. $\left({ }^{* *}\right) P<0.01 ;\left(^{*}\right) P<0.05$.

CAFs exhibited elevated expression of the major myofibroblast and contractility markers aSMA/Acta2 and MLC2/Myl9 (Fig. 1C; Supplemental Fig. S1B). Both of these genes are MRTF targets, and their expression in CAFs was MRTF-dependent (Supplemental Fig. S1B). In contrast, the TCF (ternary complex factor)-SRF target gene Egr1 was expressed at comparable levels in NFs 
Table 1. Direct MRTF-SRF target genes are overexpressed in CAFs and overlap with direct genomic targets of the YAP pathway

\begin{tabular}{|c|c|c|c|}
\hline \multicolumn{2}{|r|}{$\begin{array}{l}\text { Overlap between MRTF stringent gene signatures (683 genes) (Esnault et al. 2014) and gene } \\
\text { signatures }\end{array}$} & \multirow{2}{*}{$\begin{array}{c}\begin{array}{c}\text { Number of genes } \\
\text { (total) }\end{array} \\
31(251)\end{array}$} & \multirow{2}{*}{$\frac{P \text {-value }}{3.68 \times 10^{-9}}$} \\
\hline A & $\begin{array}{l}\text { Overexpressed in CAFs associated with MMTV-PyMT invasive carcinoma (Calvo et al. } \\
\text { 2013) }\end{array}$ & & \\
\hline & $\begin{array}{l}\text { Overexpressed in myCAFs (myofibroblastic CAFs) associated with PDAC (Ohlund et al. } \\
\text { 2017) }\end{array}$ & $39(591)$ & $6.78 \times 10^{-4}$ \\
\hline \multirow[t]{4}{*}{ B } & Induced by YAP expression in mouse livers (Dong et al. 2007) & $86(1485)$ & $6.25 \times 10^{-5}$ \\
\hline & Induced by YAP expression in MCF10A (Zhao et al. 2008) & $86(1567)$ & $4.39 \times 10^{-4}$ \\
\hline & YAP-induced in MCF10A (Zhang et al. 2009a) & $91(1240)$ & $1.36 \times 10^{-9}$ \\
\hline & YAP S127A-induced in myoblasts (Judson et al. 2012) & $54(501)$ & $7.64 \times 10^{-13}$ \\
\hline \multirow[t]{2}{*}{$\mathrm{C}$} & YAP signature (Dupont et al. 2011) & $16(93)$ & $2.35 \times 10^{-7}$ \\
\hline & YAP conserved signature (Cordenonsi et al. 2011) & $12(57)$ & $6.85 \times 10^{-7}$ \\
\hline $\mathrm{D}$ & YAP ChIP, YAP-dependent in MDA-MB231 (Zanconato et al. 2015) & $25(379)$ & 0.01 \\
\hline $\mathrm{E}$ & $\begin{array}{l}\text { Linked YAP ChIP in IMR90 lung fibroblasts (adapted from Stein et al. } 2015 \text { and Jin et al. } \\
\text { 2013) }\end{array}$ & $78(776)$ & $1.40 \times 10^{-16}$ \\
\hline \multicolumn{2}{|r|}{$\begin{array}{l}\text { Overlap between genes overexpressed in CAFs associated with MMTV-PyMT invasive } \\
\text { carcinoma ( } 302 \text { genes) (Calvo et al. 2013) }\end{array}$} & $\begin{array}{l}\text { Number of genes } \\
\text { (total) }\end{array}$ & $P$-value \\
\hline \multirow{2}{*}{$\mathrm{F}$} & $\begin{array}{l}\text { Linked YAP ChIP in IMR90 lung fibroblasts (adapted from Stein et al. } 2015 \text { and Jin et al. } \\
\text { 2013) }\end{array}$ & & \\
\hline & Closest transcription start site YAP ChIP, YAP-de & $8(70)$ & $\begin{array}{l}3.08 \times 10 \\
9.08 \times 10^{-7}\end{array}$ \\
\hline G & YAP ChIP, YAP-dependent in MDA-MB231 (Zanconato et al. 2015) & $7(379)$ & ns \\
\hline
\end{tabular}

Numbers of genes in common between the indicated gene sets were determined, with statistical significance estimated using the Bonferroni correction for multiple comparisons. (A-E) Overlap between the MRTF-SRF stringent target gene set (Esnault et al. 2014) and the following gene signatures. (A) Genes overexpressed in PyMT tumor-associated CAFs based on pooled gene expression profiles of four CAFs (Calvo et al. 2013) or PDAC-associated myCAFs (Ohlund et al. 2017). (B) Genes induced by overexpression of YAP in various contexts (Dong et al. 2007; Zhao et al. 2008; Zhang et al. 2009b; Judson et al. 2012). (C) Deduced YAP-dependent gene signatures (Cordenonsi et al. 2011; Dupont et al. 2011). (D) YAP-TEAD direct targets in MDA-MB231 cells (Zanconato et al. 2015). (E) YAP-TEAD direct targets defined by ChIP-seq and Hi-C (chromosome capture followed by high-throughput sequencing) in IMR90 lung fibroblasts (Jin et al. 2013; Stein et al. 2015). (F,G) Overlap between genes overexpressed in PyMT-associated CAFs (Calvo et al. 2013) and YAP-TEAD direct target in IMR90 lung fibroblasts and SF368 cells (F) (Jin et al. 2013; Stein et al. 2015) or YAP-TEAD direct targets in MDA-MB231 cells (G) (Zanconato et al. 2015).

and CAFs (Supplemental Fig. S1C). Consistent with these observations, ChIP analysis demonstrated increased MRTF-A, SRF, and RNA polymerase II ( $\mathrm{Pol}$ II) recruitment at Acta2 but not Egr1 (Supplemental Fig. S1E).

Previous studies have shown that MRTF activation in serum-stimulated fibroblasts is RhoA-dependent and reflects depletion of the G-actin pool as a result of formin or LIM kinase activation (Sotiropoulos et al. 1999; Tominaga et al. 2000). Consistent with this, in CAFs, the elevated activity of Acta2 and the transfected MRTF-SRF reporter was blocked when Rho was inactivated by C3 transferase expression; upon depolymerization of F-actin by latrunculin B (LatB); by the LIMK inhibitor LIMKi3; and by the formin inhibitor SMIFH2 (Fig. 1D). Acta2 and reporter expression was also inhibited by treatment with the Rho-actin inhibitor CCG-203971 (Johnson et al. 2014; Supplemental Fig. S1D). These inhibitor treatments also reduced MRTF and SRF recruitment to Acta2 as assessed by ChIP (Fig. 1E; Supplemental Fig. S1E).

The establishment and maintenance of the activated state of CAFs and myofibroblasts are promoted by substrate stiffness (for reviews, see Dupont 2016; Kalluri 2016) and TGF $\beta$ signaling (Huang et al. 2012; O'Connor et al. 2015), so we examined the role played by these stimuli in maintaining MRTF activation in CAFs. While MRTF-A remained nuclear when CAFs were plated on stiff $(50-\mathrm{kPa})$ hydrogel substrates, it relocalized to the cy- toplasm upon plating on soft $(0.5-\mathrm{kPa})$ hydrogel (Fig. 1B; Supplemental Fig. S1F). Consistent with this, treatment of CAFs with Y27632 or blebbistatin, which impair actomyosin contractility, inhibited both Acta2 expression and MRTF ChIP at Acta2 (Fig. 1D,E). TGF $\beta$ signaling was elevated in CAFs, as assessed by levels of phosphorylated Smad2 (Supplemental Fig. S2A). Treatment of CAFs with the TGF $\beta$ family receptor kinase inhibitor SB-431542 abolished Smad2 phosphorylation and induced relocalization of MRTF-A to the cytoplasm (Supplemental Fig. S2B, C). SB-431542 treatment also reduced expression of the MRTF-SRF reporter gene and the MRTF-SRF target genes Acta2 and Myl9 (Fig. 1D; Supplemental Fig. S2C,D). Conversely, in NFs, TGF $\beta$ treatment induced MRTF-A nuclear accumulation (Supplemental Fig. S2E) and activated Acta2 and Myl9 in an MRTF-dependent fashion (Supplemental Fig. S2F). Taken together, these results establish that MRTF activation is a marker of the CAF activated state and show that TGF $\beta$ signaling and contractility are required to maintain it.

The MRTFs are required for CAF contractility and matrix remodeling activity

We next tested the role of MRTF-SRF activity in matrix remodeling behavior and contractility, hallmarks of CAF function (Kalluri 2016). As a result of their increased 
ECM remodeling activity, when embedded in collagen gels, CAFs greatly facilitate matrix invasion by $4 \mathrm{~T} 1$ carcinoma cells (Gaggioli et al. 2007). Strikingly, the proinvasive activity of CAFs was substantially impaired upon siRNA-mediated depletion of the MRTFs (Fig. 2A), as was their ability to contract collagen matrices (Fig. 2B). CAF contractility was also inhibited by SMIFH2 and LIMKi3, which inhibit F-actin assembly and MRTF activation; the MRTF inhibitor CCG-203971; and SB431542 (Fig. 2C). Thus, MRTF activation is required for the proinvasive and contractile properties of CAFs.

NFs were not contractile, but their contractility was activated by serum stimulation, and this was MRTF-dependent (Fig. 2D). Expression of a constitutively active derivative of MRTF-A, MRTF ${ }^{123-1 A}$, was also sufficient to induce contractility (Fig. 2E). Previous studies have shown that the enhanced contractility and matrix remodeling activity of CAFs are dependent on the transcriptional coactivator YAP, depletion of its relative TAZ having no effect in this context (Calvo et al. 2013). Consistent with this, we found that expression of the constitutively active YAP derivative 5SA-YAP was also sufficient to induce contractility in NFs (Fig. 2E). Strikingly, however, siRNA depletion experiments showed that MRTF ${ }^{123-1 \mathrm{~A}}$. induced contractility was dependent on YAP and vice versa (Fig. 2E). Taken together, these data suggest that the enhanced contractility and proinvasive character of CAFs requires both MRTFs and YAP and that their activities are mutually dependent.

\section{Definition of candidate MRTF- and YAP-specific direct target gene sets}

Analysis of the relationship between MRTF- and YAP-dependent gene expression requires the definition of direct genomic targets for each pathway. Ideally, such definition requires identification of genomic binding sites for the factor concerned and a demonstration that transcription of genes that are in close proximity to these sites is dependent on the factor itself or the signal pathway that regulates it. Previously, we used this approach to define MRTF-SRF target genes in mouse NIH3T3 fibroblasts based on transcriptional response to MRTF-linked signals and proximity to MRTF-SRF genomic binding sites (Esnault et al. 2014; Gualdrini et al. 2016). The MRTFSRF gene signature overlaps significantly with genes overexpressed in CAFs (Table 1A) as well as genes induced by YAP overexpression in different cell types (Table 1B,C; Dong et al. 2007; Zhao et al. 2008; Zhang et al. 2009a; Cordenonsi et al. 2011; Dupont et al. 2011; Judson et al. 2012).

Recent ChIP-seq studies have identified genomic binding sites for YAP-TEAD in normal and transformed human cells (Stein et al. 2015; Zanconato et al. 2015), many of which are associated with genes that are MRTF-SRF targets in fibroblasts (Table 1D; Esnault et al. 2014; Gualdrini et al. 2016). We further refined this analysis by integrating the IMR90 ChIP-seq data with an IMR90 Hi-C (chromosome capture followed by high-throughput sequencing) data set (Jin et al. 2013) to identify genes whose transcription start sites (TSSs) are in physical contact with YAP-TEAD-binding sites. This analysis defined 776 potential YAP-TEAD direct targets in IMR90 fibroblasts (Supplemental Table S2B). Both the MRTF-SRF and the YAP-TEAD target gene sets are enriched for cytoskeletal and ECM remodeling genes and many genes involved in proliferation, signaling, and transcription (Supplemental Table S3). Many were also overexpressed in CAFs from the mouse PyMT mammary and KRas/p53 pancreatic cancer models (Calvo et al.
A

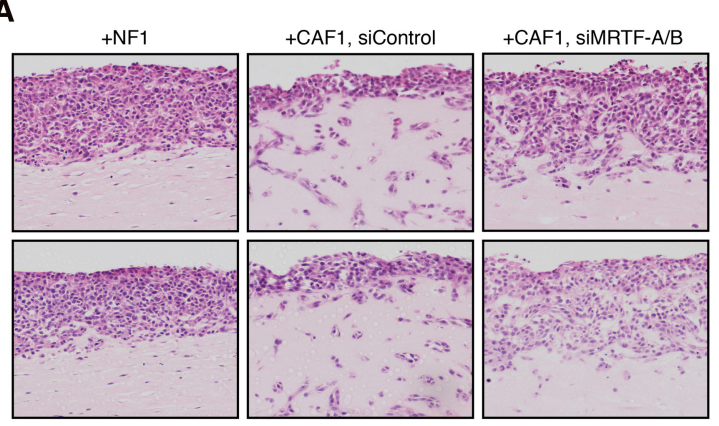

B

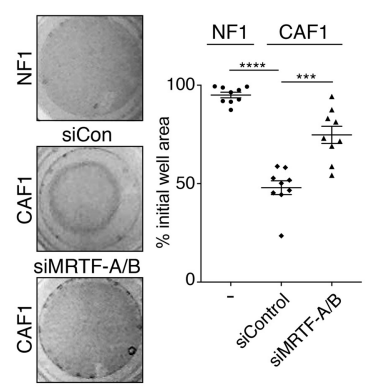

C

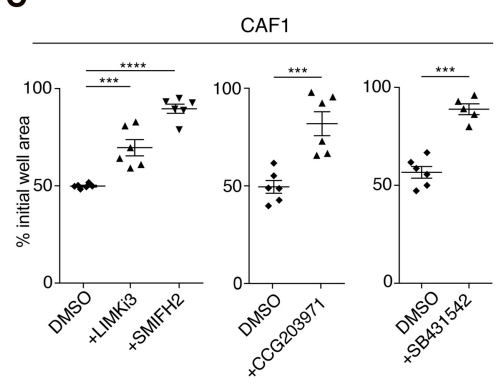

D

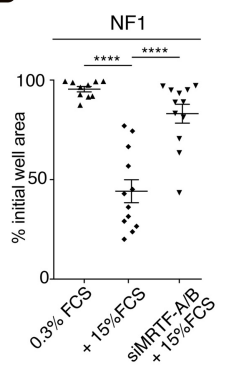

Figure 2. MRTFs are required for CAF matrix remodeling activity and contractility. (A) Representative images of invasion of 4T1 breast carcinoma cells into collagenMatrigel containing NF1 or CAF1 cells treated with control or MRTF-A/B siRNA as indicated. $(B, C)$ MRTF activity is required for contractility of CAF1 cells plated in collagen-Matrigel. $(B)$ Cells were treated for $72 \mathrm{~h}$ with MRTF-A/B siRNA. $n=3$, each plated into three gels. $(C)$ Cells were pretreated for $20 \mathrm{~h}$ with inhibitors as indicated before plating. $n=3$, each plated into two gels. $(D, E)$ Normal fibroblasts exhibit MRTF- and YAP-stimulated contractility. (D) NF1 cells with siMRTF-A/B pretreatment as indicated were stimulated with $15 \%$ FCS. $n=4$, each plated into four gels. (E) Cells transfected with constitutively active MRTF $^{123-1 A}$ or 5SA-YAP expression plasmids with siMRTF-A/B or siYAP as indicated. $n=3$, each plated into four gels. Data are mean contraction at $24 \mathrm{~h} \pm$ SEM. $\left(^{* * * *}\right) P<0.0001 ;\left(^{* * *}\right) P<0.001, t$-test. 
2013; Ohlund et al. 2017) and from human pancreatic, oral, and breast carcinoma (Table 1F, G; Supplemental Tables S4-S7; Farmer et al. 2009; Lim et al. 2011; Moffitt et al. 2015; Ohlund et al. 2017).

\section{MRTF- and YAP-specific direct target genes are activated in CAFs}

Many of the YAP-TEAD target genes in human IMR90 cells were identified previously as direct MRTF-SRF target genes in mouse NIH3T3 cells (Table 1E; Supplemental Table S2E). Comparison of the two data sets thus allowed us to identify potential candidate direct target genes specific for MRTF-SRF alone, specific for YAPTEAD alone, or shared by both regulators (referred to here as "MRTF-only," "YAP-only," and "shared" targets) (Supplemental Table S2C-E). All MRTF-only direct genomic targets analyzed were expressed at high levels in CAFs compared with NFs (Fig. 3A); we note that many of these were not detected as elevated in the previous analysis (Calvo et al. 2013), presumably because of the relatively insensitive Illumina array technique used. Similarly, expression of shared MRTF-SRF/YAP-TEAD genomic targets was also elevated in CAFs compared with NFs (Fig. $3 \mathrm{~B}$ ), as was expression of candidate YAP-only genomic targets (Fig. 3C). Strikingly, however, MRTF depletion impaired not only the expression of the MRTF-only genomic targets but also the YAP-only targets (Fig. 3D), and, conversely, YAP depletion impaired expression of MRTF-SRF genomic targets regardless of whether they contain YAP-TEAD-binding sites (Fig. 3E). Thus, MRTF-SRF signaling and YAP-TEAD signaling are mutually dependent.

\section{MRTF-SRF activity is dependent on YAP-TEAD and vice versa}

The mutual dependence of MRTF-SRF and YAP-TEAD target gene expression even at those targets that are directly bound by only one of the transcription factor pairs strongly suggests that their mutual dependence reflects the influence of each on the other's cognate signaling pathway. To examine their mutual dependence more directly, we studied the regulation of MRTF-SRF and YAP-TEAD reporter genes. The elevated activity of an MRTF-SRF reporter in CAFs was sensitive to depletion of not only the MRTFs or SRFs and the Rho-actin inhibitor CCG-203971 (Fig. 4A) but also both YAP and its DNAbinding cofactor, TEAD1 (Fig. 4B). Similarly, a YAPTEAD reporter also exhibited elevated activity in CAFs, which was dependent both on YAP and TEAD and on MRTF and SRF and was inhibited by CCG-203971 (Fig. $4 \mathrm{C}, \mathrm{D})$. Furthermore, MRTF and YAP were also mutually dependent in NFs. Serum-stimulated activity of the MRTF-SRF reporter was partially inhibited by depletion of YAP (Supplemental Fig. S3A), as was induction of the MRTF-SRF direct target genes Acta2 and Tagln (Supplemental Fig. S3B,C). Conversely, the modest response to serum stimulation of the YAP-TEAD reporter and the
A

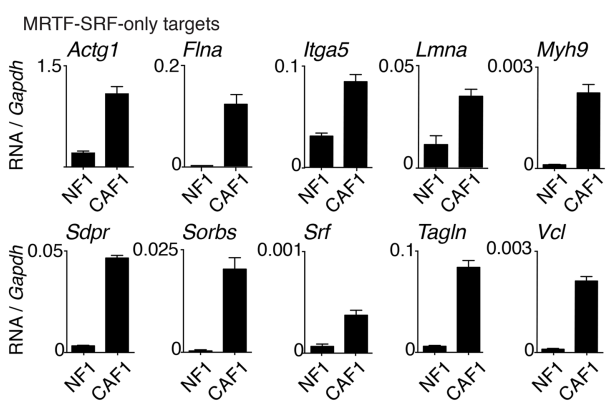

B

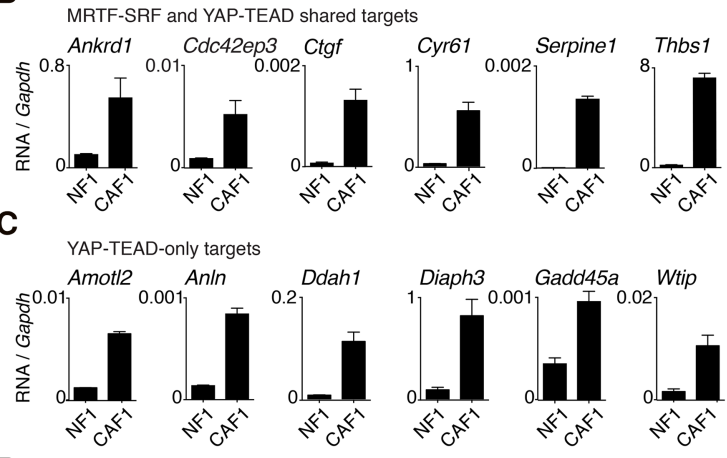

D

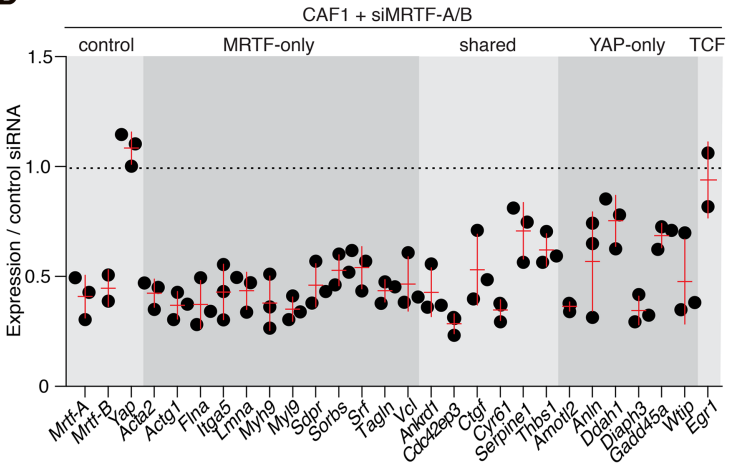

E

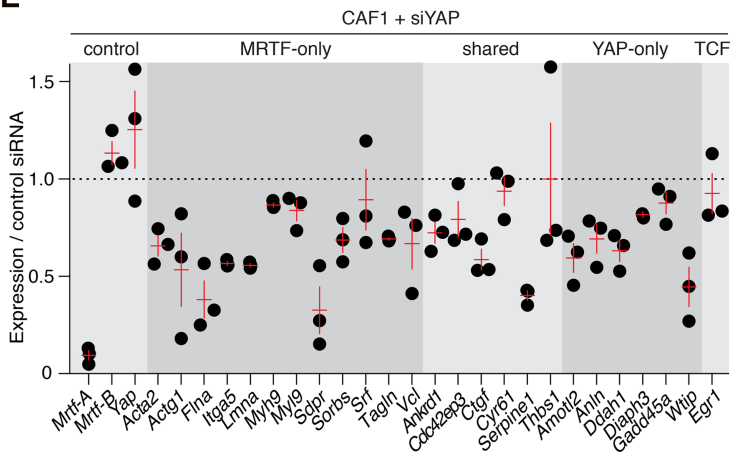

Figure 3. MRTF and YAP direct target genes are activated in CAFs. $(A-C)$ Quantitative PCR (qPCR) analysis of MRTF and YAP target gene expression in NF1 and CAF1. Data are mRNA normalized to Gapdh transcripts. Mean \pm SEM. $n=3$. (A) MRTF-only targets. (B) Shared MRTF/SRF and YAP/TEAD targets. $(C)$ YAP-only targets. $(D, E)$ Mutual dependence of MRTF and YAP target gene expression. RNA from CAF1 cells was treated with siRNA against MRTF-A/B $(D)$ or $\mathrm{YAP}(E)$. Data points represent independent siRNA treatments normalized to the geometric mean from triplicate control siRNA treatments. Red lines indicate mean and SEM. 
A

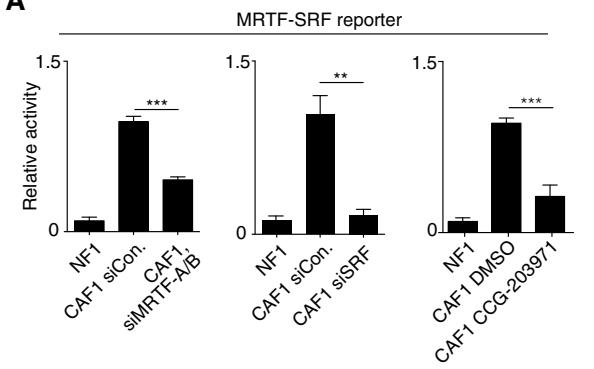

B

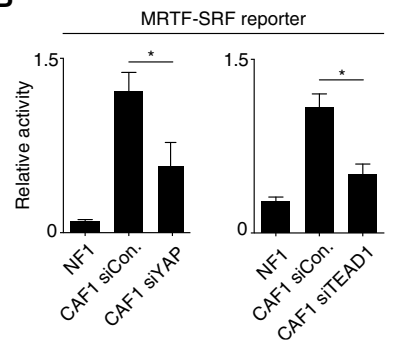

C

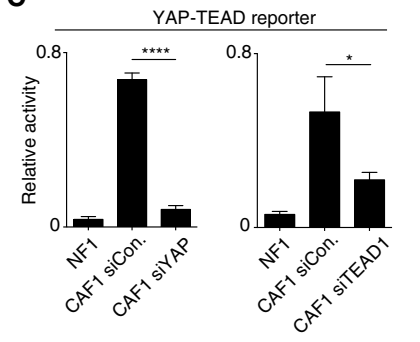

D

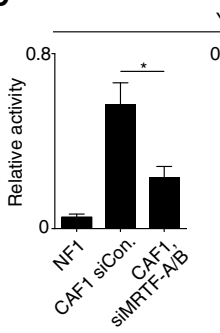

E

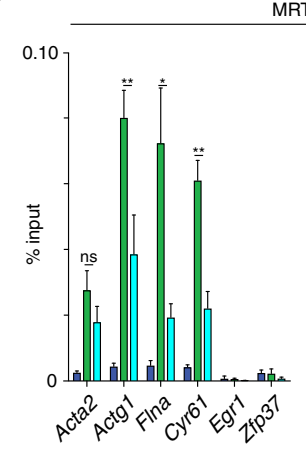

MRTF-A ChIP

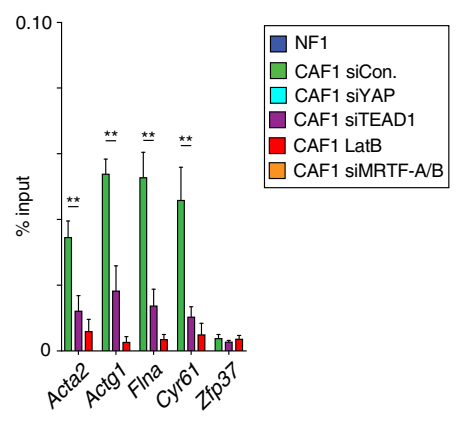

YAP-TEAD reporter

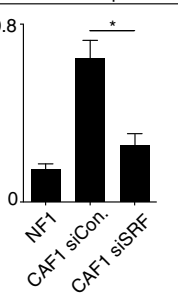

$\mathbf{F}$

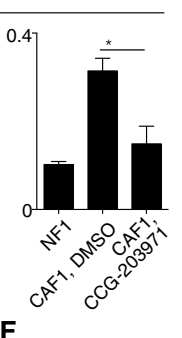

TEAD1 ChIP

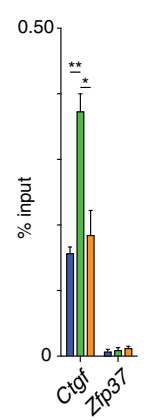

Figure 4. MRTF-SRF reporter gene activity is indirectly dependent on YAP-TEAD function and vice versa. NF1 or CAF1 cells were depleted of MRTFs, SRFs, YAP, or TEAD and transfected with the MRTF-SRF or YAP-TEAD reporters with CCG203971 treatment and serum stimulation as indicated. Data are means \pm SEM. $n=4 .(* * *) P<0.0001$; $\left.\left({ }^{* * *}\right) P<0.001 ;{ }^{* *}\right) P<0.01 ;\left({ }^{*}\right) P<0.05$, by $t$-test. $(A$, $B)$ Elevated MRTF-SRF reporter activity in CAFs. $(C, D)$ Elevated YAP-TEAD reporter activity in CAFs. Note that YAP knockdown alone is sufficient to reduce reporter activity to baseline, suggesting that TAZ does not play a significant role in this system, consistent with previous functional analysis (Calvo et al. 2013). (E,F) ChIP analysis of MRTF-A binding $(E)$ and TEAD1 binding $(F)$. Controls were Egr1 (TCF-SRF target, no MRTF-binding) and Zfp37 (no SRF or TEAD binding). Cells were treated with siRNAs or LatB as indicated. $\left.\left.{ }^{* *}\right) P<0.01 ;{ }^{*}\right) P<$ 0.05 , by $t$-test.
YAP-TEAD-specific direct target genes Amot12 and Ddah1 was inhibited by depletion of both MRTFs and YAP (Supplemental Fig. S3D-F).

We used ChIP to investigate whether the reporter results reflected changes in recruitment of MRTF and YAP to target genes. The enhanced recruitment of MRTF to MRTF-SRF target genes was enhanced in CAFs compared with NFs (Fig. 4E). It was significantly reduced upon depletion of either YAP or its DNA-binding partner protein, TEAD1, suggesting that MRTF activity is dependent on YAP-TEAD target gene expression (Fig. 4E). Inadequate antibodies precluded analysis of recruitment of YAP to YAP-TEAD targets in CAFs, but binding of its DNA-binding partner, TEAD1, was increased in CAFs compared with NFs and sensitive to depletion of MRTF (Fig. 4F). These data suggest that YAP-TEAD binding is MRTF-dependent. We confirmed this using human MDA-MB231 breast carcinoma cells, which are also migratory and invasive and display nuclear MRTF (Medjkane et al. 2009). Here, ChIP analysis revealed specific recruitment of MRTF-A to SRF sites and of YAP to TEAD sites; again, binding of MRTF was YAPdependent and vice versa (Supplemental Fig. S4). Taken together, these results suggest that the mutual dependence of MRTF and YAP activity arises indirectly and is likely to reflect their influence on each other's signal pathway.

\section{MRTF and YAP contribute independently to target gene regulation in NFs and CAFs}

Recent studies have demonstrated physical interaction between MRTF and YAP independent of their DNA-binding cofactor proteins, SRF and TEAD (Speight et al. 2016; Yu et al. 2016; Kim et al. 2017). The ChIP data presented above and the fact that YAP-TEAD and MRTF-SRF ChIPseq data exhibit only partial overlap suggest that YAPMRTF interaction is not sufficient to explain the mutual dependence of the two systems. Nevertheless, we sought to demonstrate directly that the two pathways are independently regulated, focusing on their response to the actin-binding drugs cytochalasin $\mathrm{D}(\mathrm{CD})$ and LatB. CD directly activates the MRTFs by blocking their inhibitory interaction with G-actin, while LatB inhibits MRTF activity by increasing cellular G-actin level (Miralles et al. 2003; Vartiainen et al. 2007), but both agents inhibit YAP by inhibiting F-actin assembly (Wada et al. 2011; Yu et al. 2012).

In CAFs, LatB treatment resulted in the redistribution of both factors to the cytoplasm; in contrast, while CD 
treatment induced cytoplasmic localization of YAP, it potentiated nuclear accumulation of MRTFs (Fig. 5A,B). Consistent with this, the different classes of MRTF and YAP target genes responded differentially to CD treatment. In normal fibroblasts, MRTF-only and shared targets were CD-inducible, while YAP-only targets were not (Fig. 5C; Supplemental Fig. S5). In CAF1 cells, activity of MRTF-only targets was similar to that seen in CDtreated NFs and was further potentiated by CD (Fig. 5C; Supplemental Fig. S5A). The basal activity of shared MRTF-SRF/YAP-TEAD target genes in CAFs was higher than in CD-stimulated NFs, and CD stimulation had a modest or no further effect (Fig. 5C, middle; Supplemental Fig. S5B). In contrast, although YAP-only target genes were expressed at elevated levels in CAFs, their transcription was suppressed by CD treatment (Fig. 5C; Supplemental Fig. S5B).

Taken together, these results suggest that MRTF recruitment and YAP recruitment to DNA are independently regulated. Although our inability to detect mouse YAP by ChIP precluded a direct test of this model using CAFs, ChIP analysis in the human MDA-MB231 cell system corroborated the transcription data. At all target types, MRTF and YAP ChIP signals were abolished by LatB (Fig. 5D,E).
However, CD treatment increased recruitment of MRTF and SRF to target genes (Fig. 5D) but decreased binding of YAP and TEAD (Fig. 5E). Thus, although the two factors can physically interact, their recruitment to DNA is independently regulated, and, at shared targets, expression levels represent the integration of separate signals at discreet MRTF-SRF- and YAP-TEAD-binding sites.

\section{Constitutively active MRTF and YAP derivatives are not mutually dependent}

The data presented so far suggest that the mutual dependence of MRTF and YAP may reflect their ability to influence each other's upstream signal pathways, both of which involve alterations in cytoskeletal dynamics. To test this idea, we asked whether constitutively active derivatives of MRTF and YAP, whose activity is uncoupled from upstream signal pathways, are mutually dependent. MRTF $^{123-1 A}$ is nuclear and active because it cannot bind G-actin (Vartiainen et al. 2007), while 5SA-YAP lacks the phosphorylation sites required for its cytoplasmic retention by 14-3-3 protein (Wada et al. 2011; Zhao et al. 2012).

In normal fibroblasts, expression of $\mathrm{MRTF}^{123-1 \mathrm{~A}}$ effectively activated an MRTF-SRF reporter gene and MRTF-
A

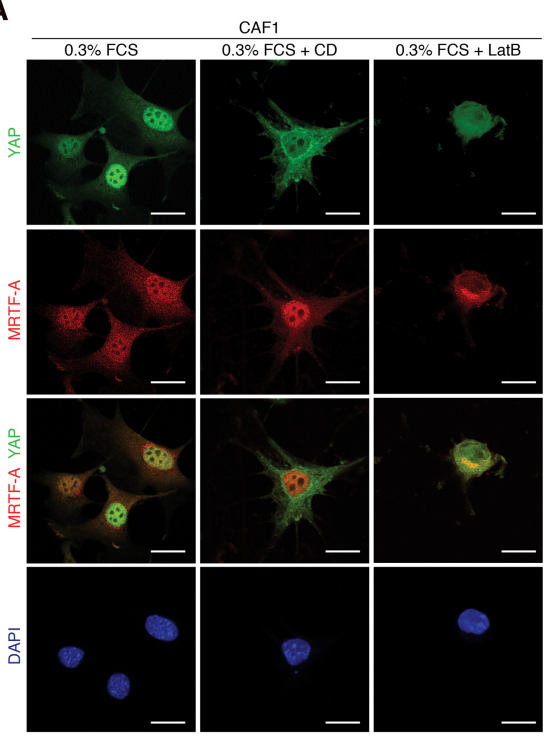

D

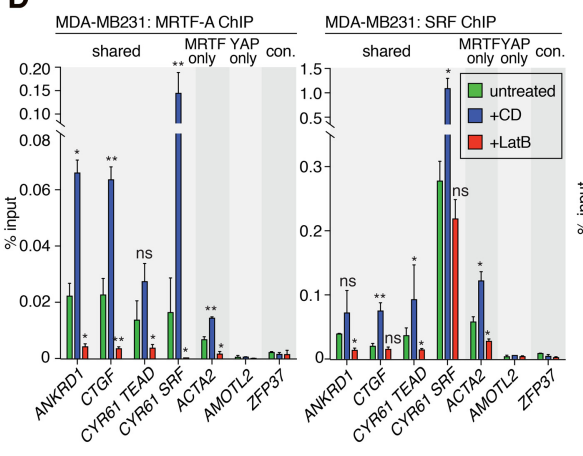

B

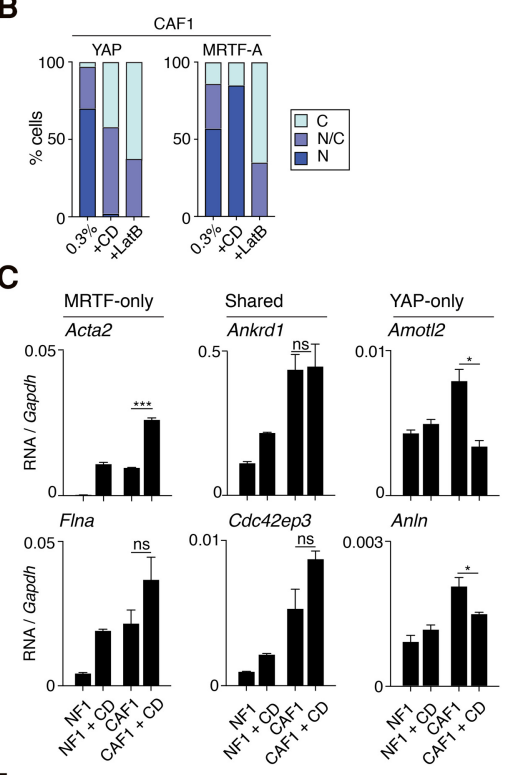

E

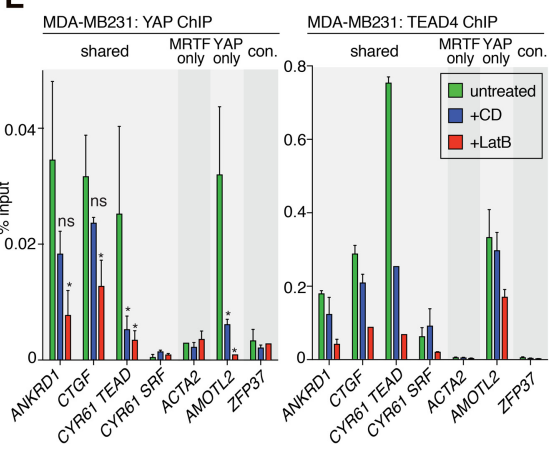

Figure 5. MRTF and YAP are independently regulated. $(A, B)$ Immunofluorescence analysis of MRTF-A and YAP in cells treated with $2 \mu \mathrm{M} \mathrm{CD}, 1 \mu \mathrm{M}$ LatB, or DMSO vehicle for $30 \mathrm{~min}$. (A) Representative images. Bar, $20 \mu \mathrm{m}$. (B) Quantitation of YAP and MRTF-A subcellular localization from 20 fields of view at $20 \times$ magnification. (C) High cytoplasmic concentration; (N/C) equal concentration over the whole cell; (N) high nuclear concentration. $(C)$ qPCR analysis of MRTF and YAP target gene intronic RNA in NF1 and CAF1 cells stimulated with $\mathrm{CD}$ for $30 \mathrm{~min}$. Data are means \pm SEM. $n=3$. (Left) MRTF-only target genes. (Middle) Shared targets. (Right) YAP-only targets. (D) ChIP analysis of MRTF-SRF and YAP-TEAD recruitment to target genes in MDA-MB231 cells treated with CD or LatB for $30 \mathrm{~min}$, as indicated. For target gene details, see Supplemental Figure S4. The Zfp37 control does not bind either MRTF-SRF or YAP-TEAD. $(D)$ MRTF-A and SRF binding. (E) YAP and TEAD4 binding. Data are means \pm SEM. $n$ $=3$ independent chromatin preparations. $\left(^{* *}\right) P<0.01 ;\left(^{*}\right) P<0.05$ Student's $t$-test, relative to untreated. The sensitivity of SRF binding to drug treatments likely reflects cooperative MRTF-SRF recruitment to promoters (Esnault et al. 2014; Gualdrini et al. 2016); the sensitivity TEAD4 binding to drug treatment is consistent with the YAP dependence of TEAD binding observed previously by others (Stein et al. 2015) and may reflect either cooperative recruitment or nuclear export of TEAD4. 
only target genes independently of YAP, consistent with a model in which transcription activation by MRTF does not require direct recruitment of YAP (Fig. 6A; Supplemental Fig. S6A). Activation of both MRTF-SRF and YAP-TEAD reporters by MRTF $^{123-1 A}$ was dependent on SRF (Fig. 6C), and both reporters could also be activated by expression of the constitutively active SRF derivative SRF-VP16 independently of MRTF (Fig. 6D). In contrast, MRTF $^{123-1 \mathrm{~A}}$ also activated the YAP-TEAD reporter gene and endogenous YAP-TEAD targets, but this was YAP-dependent (Fig. 6A,B; Supplemental Fig. S6B). Together, these data strongly suggest that $\mathrm{MRTF}^{123-1 \mathrm{~A}}$ activates the YAP-TEAD pathway by inducing MRTF-SRF target gene expression.

Similarly, although the constitutively active YAP derivative 5SA-YAP activated both the YAP-TEAD reporter and YAP-TEAD target genes independently of MRTF (Fig. 6E,F; Supplemental Fig. S7A), its ability to activate the MRTF-SRF reporter was dependent on TEAD (Fig. 6G; Supplemental Fig. S7B). In contrast, activation of shared target genes by MRTF ${ }^{123-1 A}$ and 5SA-YAP was par- tially dependent on YAP and MRTF, respectively (Fig. 6H; Supplemental Figs. S6C, S7C). These data are consistent with our assignment of the various target genes to MRTF-only, YAP-only, and shared classes. Moreover, they suggest that constitutively active MRTF activates YAP upstream signal pathways as a result of its ability to activate MRTF-SRF target gene expression and vice versa.

\section{MRTF-SRF signaling activates YAP through cell contractility}

We next investigated the mechanistic basis for the mutual dependence of MRTF-SRF and YAP-TEAD signaling. Both pathways are responsive to the state of actin dynamics, so we first investigated whether $\mathrm{MRTF}^{123-1 \mathrm{~A}}$ or $5 \mathrm{SA}$ YAP affects F-actin assembly. Both proteins promoted Factin assembly in NFs, as judged by F-actin pelleting assays and phalloidin staining (Fig. 7A; Supplemental Fig. $\mathrm{S} 8 \mathrm{~A})$. Expression of MRTF $\mathrm{MR}^{123 \mathrm{~A}}$ in NFs induced YAP nuclear accumulation, which was inhibited upon depolymerization of F-actin by LatB (Fig. 7B). Consistent with this,
A

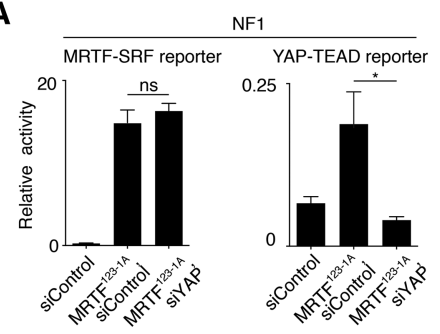

C

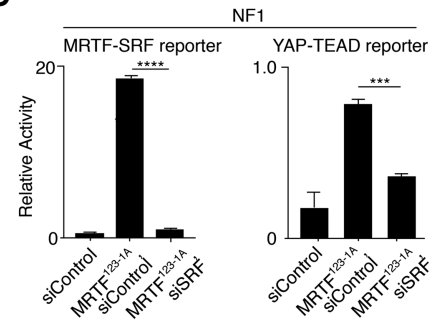

E

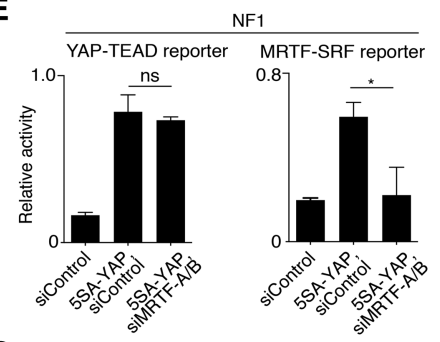

G

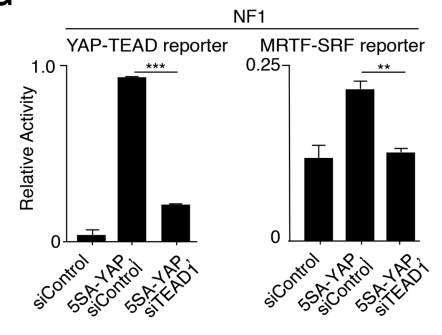

B

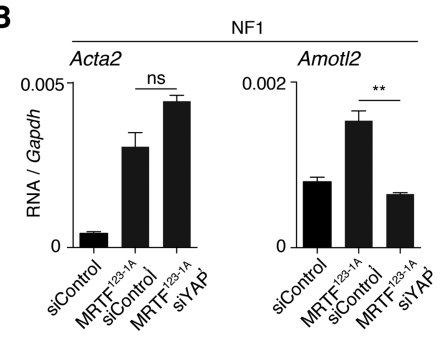

D

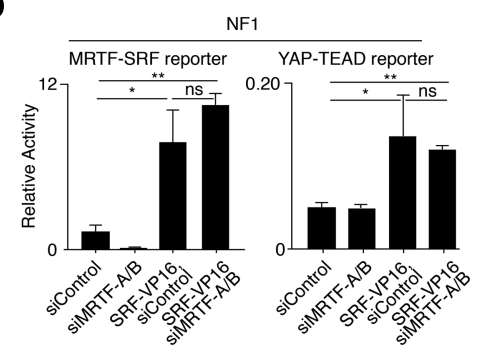

$\mathbf{F}$

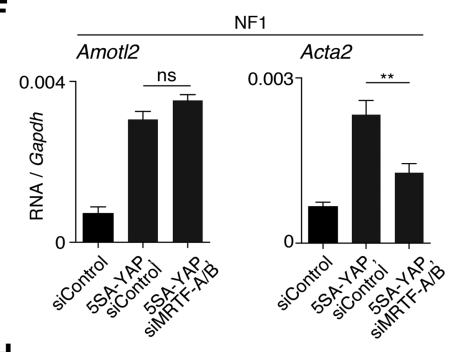

H

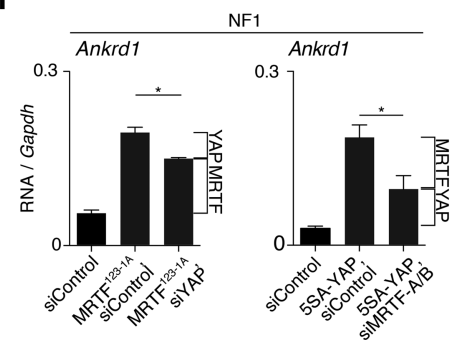

Figure 6. Constitutively active MRTF and YAP derivatives are not mutually dependent. NF1 cells were depleted of MRTFs, SRFs, YAP, or TEAD, as indicated, and transfected with the MRTF-SRF reporter or YAPTEAD reporters together with expression plasmids encoding MRTF ${ }^{123-1 A}$, SRF-VP16, or 5SA-YAP, as indicated. Data are means \pm SEM. $n=3 .\left(^{* * * *}\right) P<0.0001 ;\left(^{* * *}\right)$ $\left.P<0.001 ;\left({ }^{* *}\right) P<0.01 ;\left.\right|^{*}\right) P<0.05$, by Student's $t$-test. $(A, B)$ MRTF $^{123-1 \mathrm{~A}}$ activates the MRTF-SRF reporter $(A)$ and MRTF-SRF target genes $(B)$ independently of YAP. (C) MRTF $^{123-1 \mathrm{~A}}$ requires SRF to activate both the MRTF-SRF and YAP-TEAD reporters. (D) SRF-VP16 does not require MRTFs to activate the MRTF-SRF and YAP-TEAD reporters. $(E, F)$ 5SA-YAP activates the YAP-TEAD reporter $(E)$ and YAP-TEAD target genes $(F)$ independently of MRTFs. $(G)$ 5SA-YAP requires TEAD1 to activate both the MRTF-SRF and YAPTEAD reporters. $(H)$ Indirect YAP and MRTF signaling contributes to activation of the MRTF-SRF and YAPTEAD shared target gene Ankrd1 by constitutively active MRTF ${ }^{123-1 A}$ or 5SA-YAP. Notional contributions of YAP and MRTF activation are indicated by brackets. 
A

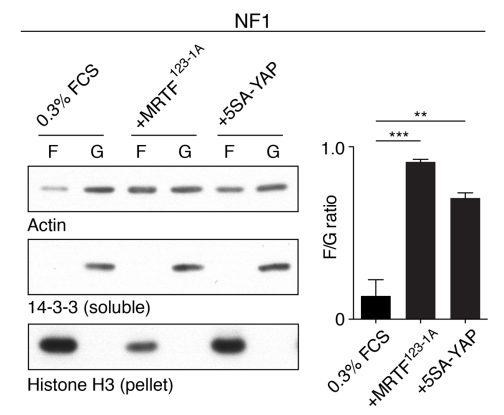

B

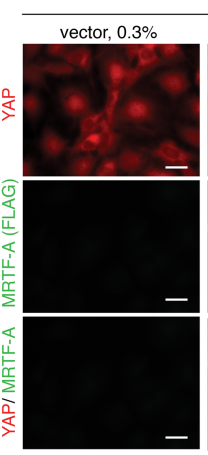

C

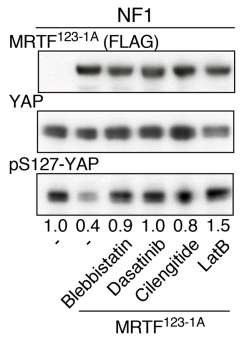

$\mathbf{F}$

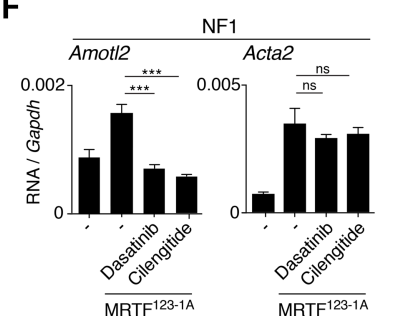

D

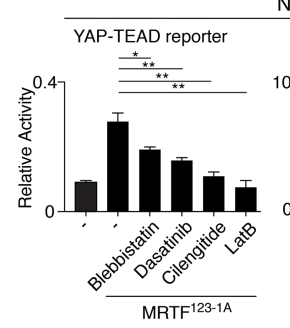

G

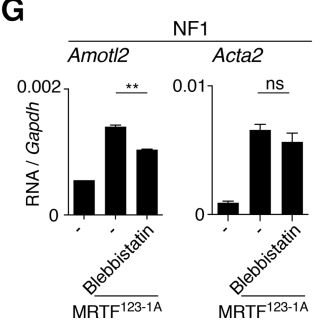

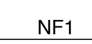

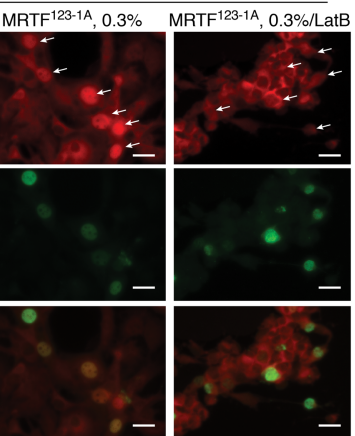

E

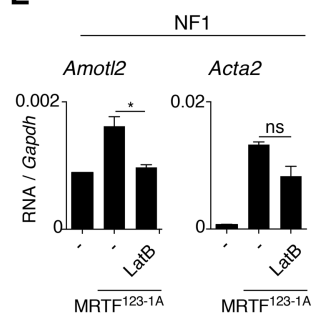

H

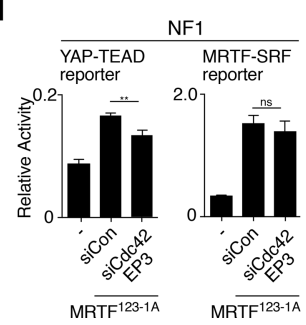

Figure 7. MRTF activates YAP through cell contractility. $(A$, left $)$ Constitutively active MRTF $^{123-1 \mathrm{~A}}$ and 5SA-YAP increase the proportion of actin in the pellet (lanes $F$ ) compared with soluble fractions (lanes $G$ ) in sedimentation assays. 14-3-3 and $\mathrm{H} 3$ are controls for soluble and pellet fractions. (Right) Quantitation. Data are means \pm SEM. $n=3 .\left({ }^{* *}\right) P<0.01$, Student's $t$-test. $(B)$ Immunofluorescence analysis of NF1 cells with or without MRTF ${ }^{123-1 \mathrm{~A}}$ expression with $30 \mathrm{~min}$ LatB treatment, as indicated. Arrows indicate cells overexpressing MRTF $^{123-1 \mathrm{~A}}$. Bar, $25 \mu \mathrm{m}$. (C) Immunoblot analysis of YAP S127 phosphorylation in NF1 cells expressing MRTF ${ }^{123-1 \mathrm{~A}}$ with 2-h inhibitor treatments, as indicated. $(D)$ Analysis of MRTF-SRF or YAP-TEAD reporter activity in cells expressing MRTF ${ }^{123-1 A}$ with inhibitor treatment, as indicated. Data are means \pm SEM. $n=3$. ( $\left.{ }^{* *}\right) P<0.01$; $(*) \quad P<0.05 . \quad(E-G) \quad$ Cells expressing MRTF $^{123-1 \mathrm{~A}}$ were treated with inhibitors as indicated, and expression of intronic RNA of the MRTF-only target Acta2 and the YAP-only target Amot12. Data are means \pm SEM. $n=3$. $\left({ }^{* * *}\right) P<0.001 ;(* *) P<$ 0.01 ; $\left.{ }^{*}\right) P<0.05$. $(H)$ Analysis of reporter activity in NF1 cells expressing MRTF ${ }^{123-1 A}$ with depletion of Cdc42EP3, as indicated. Analysis was as in $D$. in MDA-MB231 cells, MRTF ${ }^{123-1 A}$ expression promoted increased YAP ChIP at YAP targets, which was abolished by LatB (Supplemental Fig. S8C).

Cell adhesion and cell contractility act via Src kinases to activate YAP by antagonizing LATS-mediated phosphorylation of YAP S127, a major target for Hippo signaling (Kim and Gumbiner 2015; Si et al. 2017; for review, see Meng et al. 2016). Cell adhesion, actomyosin contractility, and Src kinases are also required for maintenance of active YAP in CAFs (Calvo et al. 2013). Expression of MRTF $^{123-1 A}$ in NF1 cells decreased YAP S127 phosphorylation, which was accompanied by increased Src S416/418 and MLC2 phosphorylation (Supplemental Fig. S8D). In NF1 cells, inhibition of myosin (blebbistatin), Src family kinases (dasatinib), or integrinav (cilengitide) blocked MRTF $^{123-1 \mathrm{~A}}$-induced YAP S127 dephosphorylation (Fig. 7C) and inhibited activation of the YAP reporter by MRTF $^{123-1 A}$ without affecting activation of the MRTF reporter (Fig. 7D). Activation of YAP-only and MRTF-only target genes by MRTF ${ }^{123-1 \mathrm{~A}}$ was affected in a similar manner (Fig. 7E-G).

These results show that YAP activation by MRTF $^{123-1 A}$ involves increased cell contractility, a property that is strongly dependent on MRTF-SRF target gene expression (Esnault et al. 2014; Gualdrini et al. 2016). We showed above that in NFs, MRTF ${ }^{123-1 A}$ acti- vates expression of contractile genes-including aSMA/ Acta2, MLC2/My19, and Myh9 (Fig. 6B; Supplemental Fig. S6) - and contractility itself (Fig. 2E), and, in CAFs, contractile gene expression and contractility are MRTFdependent (Fig. 2B; Supplemental Fig. S1B). Expression of integrins Itgb3 and Itgav, specific targets for cilengitide action, was elevated in CAFs and could be potentiated in NFs by MRTF ${ }^{123-1 A}$ expression (Supplemental Fig. S8D,E).

Recent studies have shown that the septin regulator Cdc42EP3 is required in CAFs for F-actin assembly, contractility, and YAP activation (Calvo et al. 2015). In CAFs, expression of Cdc42EP3, which is a direct MRTF-SRF target in fibroblasts (Esnault et al. 2014; Gualdrini et al. 2016), was elevated and MRTF-dependent (Fig. 3D), and, in NFs, it was strongly activated by MRTF ${ }^{123-1 A}$ and CD (Fig. 5C; Supplemental Fig. $\mathrm{S} 6 \mathrm{C})$. Consistent with the reported dependence of Factin assembly on Cdc42EP3 levels (Calvo et al. 2015), the ability of $M R T F^{123-1 A}$ to induce activity of the YAP-TEAD reporter and YAP-TEAD target genes in NFs was sensitive to Cdc42EP3 depletion (Fig. 7H). Taken together with the preceding results, these data show that MRTF-SRF signaling potentiates YAP activity through activation of multiple genes involved in cell contractility. 


\section{YAP-TEAD activation of MRTF requires TGF $\beta$ signaling}

Consistent with the ability of constitutively active 5SAYAP to induce F-actin assembly, it also induced nuclear accumulation of MRTF; this was inhibited by LatB and was not induced by 5SA/S94A-YAP, indicating that it involves F-actin assembly and requires activation of YAPTEAD target genes (Fig. 8A; Supplemental Fig. S9A). ChIP analysis showed that 5SA-YAP, but not 5SA/S94AYAP, expression induced LatB-sensitive MRTF-A recruitment to MRTF target genes (Fig. 8B; Supplemental Fig. S9B). 5SA-YAP expression also induced phosphorylation of MRTF-A (Supplemental Fig. S9C), which reflects decreased G-actin binding (Supplemental Fig. S8A; Panayiotou et al. 2016). Consistent with these results, LatB treatment abolished 5SA-YAP activation of the MRTF reporter and MRTF-only target genes such as Acta2 (Fig. 8C) but did not affect activation of the YAP reporter or YAPonly targets such as Amotl2 (Fig. 8D). LatB treatment of cells expressing 5SA-YAP led to immediate inhibition of transcription at MRTF-only and shared targets but not YAP-only targets (Supplemental Fig. S9D).

We showed above that the elevated MRTF activity seen in CAFs is dependent on matrix stiffness, contrac- tility, and TGF $\beta$ signaling (Fig. 1B-D; Supplemental Fig. S2A,B). Consistent with these data, the ability of 5SAYAP to potentiate MRTF-SRF reporter activity and Acta2 transcription was sensitive to the LIMK inhibitor LIMKi3, the ROCK inhibitor Y27632, and the TGF $\beta$ family receptor kinase inhibitor SB-431542, while its ability to activate the YAP-TEAD target gene Amotl2 was not (Fig. 8E; Supplemental Fig. S9E). Strikingly, elevated TGF $\beta$ signaling in CAFs, as assessed by SMAD2 S465/ S467 phosphorylation, was strongly down-regulated upon siRNA knockdown of YAP (Fig. 8F). The TGF $\beta$ component Inhba is expressed at high levels in CAFs (Supplemental Fig. S9F; Calvo et al. 2013) and mediates their autocrine dependence on TGF $\beta$ signaling (C Foster, unpubl.; D Miller and C Hill, pers. comm.). Inhba is a candidate YAP-TEAD direct target gene (Supplemental Table S2), and, in NFs, its expression was potentiated by 5SA-YAP expression (Fig. 8G).

Taken together with the results in the preceding section, these results support a model in which the potentiation of TGF $\beta$ signaling by YAP contributes to elevated MRTF-SRF activity, which in turn influences YAPTEAD signaling through potentiation of cell contractility (Fig. $8 \mathrm{H}$; see the Discussion).
A

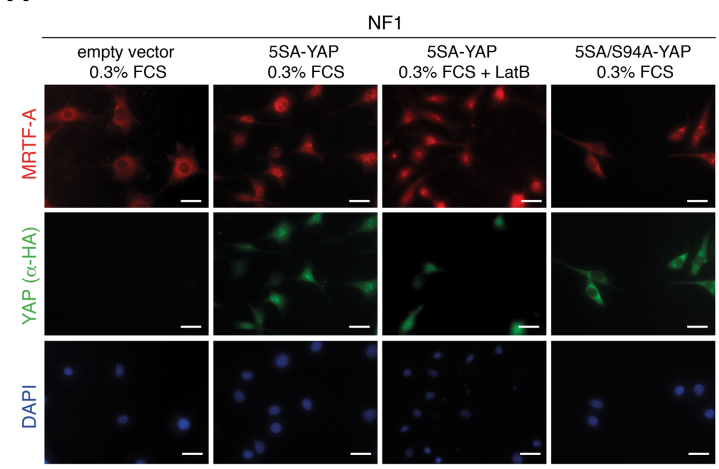

C

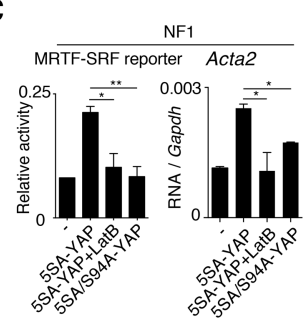

D

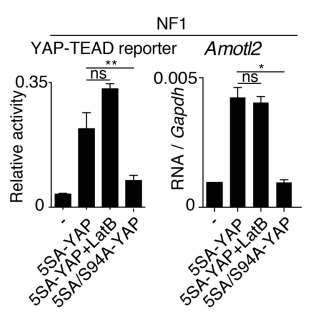

B

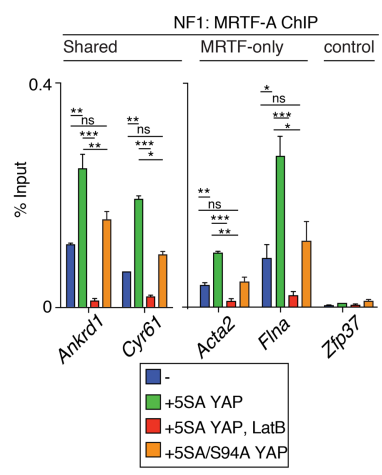

E

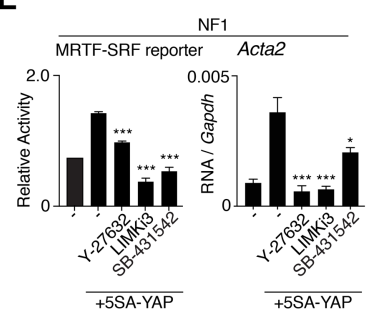

$\mathbf{F}$

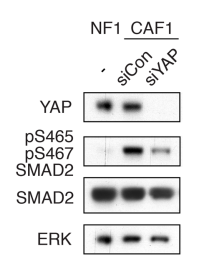

G

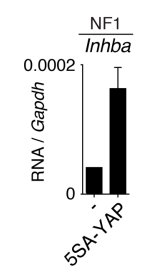

H

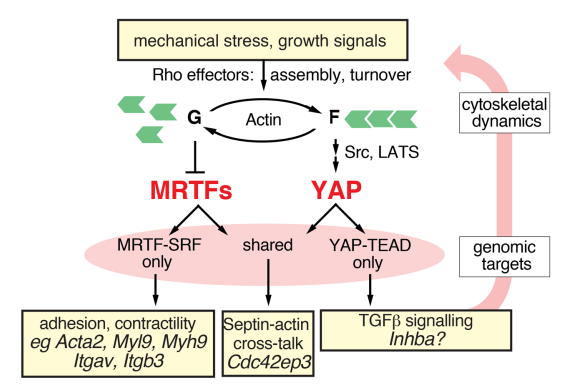

Figure 8. YAP-TEAD activation of MRTF requires TGF- $\beta$ signaling. $(A)$ Immunofluorescence analysis of NF1 cells expressing 5SA-YAP or 5SA/S94A-YAP with LatB treatment, as indicated. $(B) \mathrm{ChIP}$ analysis of NF1 cells expressing 5SA-YAP or 5SA/ S94A-YAP before or after $30 \mathrm{~min}$ of LatB treatment. Data are mean \pm SEM. $\left(^{* * *}\right) P<$ 0.001; $\left(^{* *}\right) P<0.01 ;\left(^{*}\right) P<0.05$. $(C-E)$ Cells were transfected with the MRTF-SRF reporter and 5SA-YAP or 5SA/S94A-YAP and treated with the indicated inhibitors. Data are mean \pm SEM. $n=3 .\left(^{* *}\right) P<0.01$; $\left({ }^{*}\right) P<0.05$. $(C)$ Activation of the MRTF reporter and MRTF-only target Acta2 is sensitive to LatB. (D) Activation of the YAP reporter and YAP-only target Amotl2 is not sensitive to LatB. (E) MRTF-SRF reporter activity or Acta2 expression requires Factin assembly and TGF $\beta$ signaling. $(F)$ Immunoblot analysis of YAP, S465/S467diphosphorylated Smad2, total Smad2, and ERK control in NF1 and CAF1 cells with or without depletion of YAP. $(G)$ Inhba expression levels in NF1 cells with or without 5SA-YAP expression. $(H)$ Indirect cytoskeletal cross-talk model for the mutual dependence of MRTFs and YAP. MRTF-SRF signaling influences YAP activity via mechanisms that include the potentiation of cell contractility; YAP-TEAD signaling influences MRTF activity at least in part through potentiation of TGF $\beta$ signaling. Both pathways impinge on septinactin interaction. Likely target genes involved are shown. For discussion, see the text. 


\section{Discussion}

The MRTF-SRF signaling pathway allows the coordination of gene transcription and cytoskeletal dynamics (Miralles et al. 2003; Olson and Nordheim 2010). Here we investigated the role of MRTF-SRF signaling in CAFs derived from the mouse PyMT mammary tumor model. In contrast to NFs, the MRTFs are active in CAFs and are dependent on matrix stiffness and autocrine TGF $\beta$ signaling. MRTF-SRF target gene expression is elevated and is required for CAF contractility and proinvasive properties. Strikingly, MRTF-SRF target gene expression is also dependent on the transcription coactivator YAP, which is also mechano-responsive and required for CAF proinvasive properties and vice versa even at genes that are direct targets for only one of the two pathways. MRTF and YAP can each indirectly activate the other through their ability to affect actin cytoskeletal dynamics. Our results support a model in which the mutual dependence of MRTF-SRF and YAP-TEAD arises from their ability to regulate cell contractility and TGF $\beta$ signaling (Fig. $8 \mathrm{H}$ ). YAP functions redundantly with its relative, TAZ (Meng et al. 2016). Although activity of the latter does not appear limiting in the CAF system used here (Calvo et al. 2013), our findings suggest that MRTF-SRF activity will also influence TAZdependent transcription in appropriate contexts.

We found that, in contrast to NFs, a substantial proportion of mammary CAFs associated with PyMT-induced carcinoma (Calvo et al. 2013) contains nuclear MRTF and that expression of numerous MRTF-SRF target genes is concomitantly elevated. Similarly, MRTF-SRF target gene expression is elevated in tumor-proximal myCAFs (myofibroblastic CAFs) in human pancreatic ductal carcinoma (Ohlund et al. 2017). Thus, constitutively nuclear localization of MRTF and activation of MRTF-SRF target gene expression can be considered as further CAF activation markers (for discussion, see Kalluri 2016). Constitutively nuclear MRTF has been observed previously in a number of other cell types and contexts (Somogyi and Rorth 2004; Medjkane et al. 2009) and is generally taken to be indicative of increased MRTF-SRF signaling. It should be noted, however, that nuclear confinement of the MRTFs is not necessarily sufficient for activation of MRTF-SRF target gene expression unless G-actin levels are low (Vartiainen et al. 2007).

YAP-dependent gene expression is also increased in CAFs and is required for CAF contractility and proinvasive behavior (Calvo et al. 2013). Recent ChIP-seq analysis has shown that YAP-TEAD target genes encode proteins involved in proliferation, signaling, transcription, and cytoskeletal components and regulators (Stein et al. 2015; Zanconato et al. 2015). We found that the direct YAPTEAD target gene sets contain many genes found in our previously determined MRTF-SRF gene signature (Esnault et al. 2014). These genes, which contain binding sites for both MRTF-SRF and YAP-TEAD, include several commonly used as model YAP-TEAD targets, including Cyr61, Ctgf, and Ankrd1. In line with our finding that YAP activity and MRTF activity are mutually dependent, we note that both MRTF-SRF and YAP-TEAD signatures defined here are significantly enriched in activated stromal gene expression signatures in both mouse tumor models and human breast, oral, and pancreatic cancers (Farmer et al. 2009; Lim et al. 2011; Moffitt et al. 2015; Ohlund et al. 2017).

We found that in CAFs, but not in normal fibroblasts, MRTF-A exhibited substrate stiffness-induced nuclear accumulation. Mechanical cues, including substrate stiffness, were shown previously to promote nuclear localization of YAP and TAZ (Wada et al. 2011; Calvo et al. 2013; Das et al. 2016; Dupont 2016). Application of force to integrins induces MRTF-A nuclear accumulation (Zhao et al. 2007), and substrate stiffness can activate it in lung fibroblasts and mesenchymal stem cells (Huang et al. 2012; Buxboim et al. 2014). It appears that development of the CAF phenotype involves increased sensitivity of YAP and MRTF to mechanical stimulation, but how this occurs remains unclear. In PDAC, contractile "myCAFs" are found close to the tumor, coexisting with a more distant "iCAF" (inflammatory CAF) population, and organoid experiments show that the myCAF phenotype requires physical contact with the tumor (Ohlund et al. 2017). This, together with the elevated expression of MRTF-SRF and YAP-TEAD target genes in myCAFs, suggests that the myCAF phenotype might be mechanically determined.

The MRTFs compete with the other cofactor family, the TCFs, for binding to SRFs (Miralles et al. 2003; Wang et al. 2004; Zaromytidou et al. 2006). In mouse embryonic fibroblasts (MEFs), simple deletion of all three TCFs substantially increases MRTF-dependent gene expression, resulting in greatly enhanced contractility and proinvasive behavior (Gualdrini et al. 2016). This phenotype reflects increased access of the MRTFs to SRFs rather than TCFdependent transcriptional activation and supports the notion that MRTF-SRF signaling is an important contributor to the activated CAF phenotype. However, at least at the RNA level, the relative expression of the MRTFs and TCFs did not appear to differ between NFs and CAFs.

Recent experiments have demonstrated direct physical interactions between the YAP/TAZ WW domain and a conserved PPxY motif at the $\mathrm{C}$ terminus of myocardin family proteins, including the MRTFs (Speight et al. 2016; Yu et al. 2016; Kim et al. 2017). Our experiments with constitutively activated MRTF and YAP suggest that these interactions do not directly contribute to the transactivating functions of MRTF. Moreover, they do not appear sufficient to generate a significant ChIP signal, since, even when coincident ChIP was observed, both corresponding DNA-binding partners were also detected, consistent with either the presence of closely spaced SRF and TEAD sites or looping to remote sites. We favor the idea that rather than mediating targeting, direct MRTF-YAP interaction facilitates activation of shared targets, allowing stabilization of higher-order complexes formed between activated MRTF-SRF and YAP-TEAD bound to separate DNA regulatory elements.

While both MRTF-SRF signaling and YAP-TEAD signaling are responsive to cytoskeletal state and mechanical stress, they are differentially regulated. MRTF-SRF 
signaling senses G-actin levels (Miralles et al. 2003; Vartiainen et al. 2007), whereas YAP-TEAD signaling is F-actin-dependent and sensitive to cytoskeletal tension (Dupont et al. 2011; Wada et al. 2011). Moreover, even at DNA regions where both MRTF binding and YAP binding are detected, they exhibit opposite responses to the actinbinding drug CD (Miralles et al. 2003; Vartiainen et al. 2007; Wada et al. 2011; Yu et al. 2012). Nevertheless, our results show that the pathways are mutually dependent, with inhibition (or activation) of one pathway resulting in inhibition (or activation) of the other. Our results show that this interdependence requires SRF and TEAD, the DNA-binding cofactors of MRTF and YAP, and thus must involve activation of their genomic targets. Indeed, constitutively active TEAD derivatives are sufficient to induce activation of MRTF-SRF targets such as Acta2 and Tagln (Ota and Sasaki 2008).

Cross-talk between MRTF-SRF and YAP-TEAD signaling involves multiple genes that influence cytoskeletal dynamics (Fig. 8H). YAP activation in CAFs is dependent on cell adhesion and contracility (Calvo et al. 2013, 2015). In normal fibroblasts, MRTF-SRF signaling potentiates YAP-TEAD signaling in an adhesion- and contractilitydependent manner. Major components of the contractile machinery in CAFs are MRTF-SRF targets, including Acta2, Myl9, and Myh9. MRTF-SRF signaling also potentiates expression of integrins Itgb3 and Itgav, which have been implicated previously in Src-dependent YAP activation (Kaneko et al. 2014). Both contractility and YAP activation in CAFs are also dependent on the septin regulator Cdc42ep3, an MRTF-SRF direct target gene, which was also required for cross-talk between the MRTF-SRF and YAP-TEAD pathways. Conversely, at least one route by which YAP-TEAD signaling potentiates MRTF-SRF activity appears to be through activation of autocrine TGF $\beta$ signaling, which is likely to involve elevated expression of the YAP-TEAD target Inhba. Blockade of TGF $\beta$ receptor activity restores MRTF-A cytoplasmic localization in CAFs, and TGF $\beta$ signaling has been implicated previously in the myofibroblast transition (Huang et al. 2012; O'Connor et al. 2015).

We showed that the contractile and invasiveness-promoting phenotype of CAFs reflects activation of both MRTF-SRF signaling and YAP-TEAD signaling and that these two pathways are mutually dependent. The robustness of transitions between two different functional states is substantially enhanced when they are governed by interlocking positive feedback loops, such as the simultaneous activation of a pathway promoting one state and inhibition of a pathway that inactivates that state (for discussion, see Kim and Ferrell 2007; Ferrell 2008). It is tempting to speculate that the mutual dependence of the MRTF-SRF and YAP-TEAD signal pathways contributes to the stability of the CAF activated state.

\section{Materials and methods}

Antibodies, protein detection, and immunofluorescence

The antibodies used were phospho-Smad2 (Cell Signaling Technology, 138D4), Smad2/3 (BD, 610842), SMA (Sigma, A2547),
MLC2 (Cell Signaling Technology, 3674), SRF (sc-335), phospho (T18/S19)-MLC2 (Cell Signaling Technology, 3674S), MRTF-A (sc-21558), MRTF-B (sc-47282), pan-ERK (BD, 610124), YAP (for immunofluorescence, clone 63.7, sc-101199; for ChIP, ab52771), phospho-Ser127-YAP (Cell Signaling Technology, 4911S), TEAD1/TEF-1 (BD, 610922), TEAD4/TEF3 (sc-101184), Actin (Cytoskeleton, AAN01), pan 14-3-3 (clone H-8, sc-1657), histone H3 (Abcam, ab1791); RNA Pol II CTD S5P (Covance, H14), Integrin $\beta 3$ (ab119993), Integrin $\alpha \mathrm{V}$ (ab179475), phospho-(Tyr416) Src (Cell Signaling Technology, 2101), Src (2109S), HA high affinity (3F10) (Roche, 11867431001), HA-peroxidase high affinity (3F10) (Roche, 12013819001), Flag-M2-peroxidase (Sigma, A-85292), and Flag (for ChIP, Sigma, F7425; for immunofluorescence, clone M2, F1804). Immunoblotting and immunofluorescence were by standard techniques. F-actin was visualized with Texas Red-X phalloidin (Invitrogen), and nuclei were visualized with DAPI.

\section{ChIP and RNA analysis}

ChIP was as described previously (Gualdrini et al. 2016). Three independent chromatin preparations and immunoprecipitations were analyzed in each experiment with duplicate quantitative PCR (qPCR) for each using primers as in Supplemental Table S8A. Total RNA was prepared using GeneElute (Sigma) in column format. Following DNase I treatment (Ambion), cDNA synthesis used the Transcriptor first strand cDNA synthesis kit (Roche). qPCR was performed using the ABI 7900 thermocycler (Applied Biosystems) and QuantStudio QS3/QS5 with detection by SYBR Green incorporation (Invitrogen). Relative abundance of template cDNA from intronic sequences was used as a proxy for relative transcription rates and calculated using the $\Delta \mathrm{Ct}$ method, normalizing to the abundance of GAPDH cDNA. For primers, see Supplemental Table S8B.

\section{Gel contraction, invasion, and F-actin pelleting}

Organotypic invasion assays and gel contraction assays were as described (Gualdrini et al. 2016). For inhibitor treatments and serum stimulation, cells were treated as appropriate prior to embedding and maintained in inappropriate medium during the assay. For siRNA depletions, cells were treated for $72 \mathrm{~h}$ prior to embedding. Twenty-four hours after plating, gel contraction was calculated as a percentage of well area using ImageJ. For F-actin pelleting, the F/G-actin separation kit (Cytoskeleton) was used according to the manufacturer's instructions.

\section{Cell culture, reporter assays, inhibitors, and siRNA treatments}

Immortalized NFs and CAFs from the PyMT model (Calvo et al. 2013) were maintained in $10 \%$ FCS or $0.3 \%$ FCS $+1 \%$ ITS (Invitrogen) for $20 \mathrm{~h}$ followed by stimulation with $15 \%$ serum or 2 $\mathrm{ng} / \mathrm{mL}$ TGF $\beta$. Small-molecule treatments were $10 \mu \mathrm{M}$ SB143542, $20 \mu \mathrm{M}$ CCG-203971, $1 \mu \mathrm{M}$ LatB, $2 \mu \mathrm{M}$ CD, $20 \mu \mathrm{M}$ SMIFH2, $50 \mu \mathrm{M}$ LIMKi3, $0.5 \mu \mathrm{M}$ dasatinib, and $10 \mu \mathrm{M}$ Y27632. Cells were treated for $12 \mathrm{~h}$ prior to reporter analysis and for $2 \mathrm{~h}$ prior to intronic RNA or ChIP analysis. Cells were transfected using Lipofectamine LTX and Plus reagent $(1 \mu \mathrm{L}$ of LTX/0.5 $\mu \mathrm{L}$ of Plus or $5 \mu \mathrm{L} / 2.5 \mu \mathrm{L}$ of Plus for 24 -well and six-well plates, respectively, and $30 \mu \mathrm{L}$ of LTX/15 $\mu \mathrm{L}$ of Plus or $75 \mu \mathrm{L}$ of LTX/37.5 $\mu \mathrm{L}$ of Plus for $10-\mathrm{cm}$ and $15-\mathrm{cm}$ plates). Reporter assays in 24-well plates used $20 \mathrm{ng}$ of 3DA.Luc MRTF-SRF or pGL3 4xGTIIC YAP-TEAD reporter, $5 \mathrm{ng}$ of Renilla ptk-RL control, and $100 \mathrm{ng}$ of expression plasmid with analysis by dual-luciferase reporter assay system (Promega). Expression plasmids (15 $\mu \mathrm{g}$ per $15-\mathrm{cm}$ plate) were SRF-VP16 (Miralles et al. 2003), MRTF123-1A 
(Vartiainen et al. 2007), pCMV-HA-5SA-YAP, and pCMV Flag5SA/S94A-YAP (gift from Dr Ralph Gruber). For siRNA-mediated protein depletion, cells were reverse-transfected using RNAi Max reagent with analysis $72 \mathrm{~h}$ later; expression plasmids were transfected $48 \mathrm{~h}$ prior to analysis. siRNAs $(20 \mathrm{nM})$ were mouse YAP1 (Dharmacon On-Target Plus, J-046247-10), human YAP1 (Dharmacon, L-012200), mouse TEAD1 (Dharmacon, L-048419-01), mouse Cdc42EP3 (Dharmacon, L-046421-01), mouse SRF (Dharmacon, J-050116-09), human SRF (Dharmacon M-009800), mouse MRTFA/B dual-targeting oligo 5'-UGGAGCUGGUGGAGAAGAA-3' (25 nM) (Medjkane et al. 2009), human MRTF-A (L015434), human MRTF-B (Dharmacon, M-019279), and human nontargeting pool (Dharmacon, D-001810-10-20).

\section{Bioinformatics}

Published YAP-TEAD ChIP-seq data from IMR90 lung fibroblasts (Stein et al. 2015) were integrated with a previous Hi-C analysis of IMR90 cells (Jin et al. 2013) as described previously (Gualdrini et al. 2016). The different genome alignments used in the two studies were converted to the $\mathrm{mm} 9$ assembly using the University of California at Santa Cruz LiftOver tool (https ://genome.ucsc.edu/cgi-bin/hgLiftOver). The final analysis revealed 725 TSSs that show physical linkage to remote YAPTEAD sites that, together with 65 TSSs located within $2 \mathrm{~kb}$ of a YAP-TEAD site, give a total of 776 potential YAP-TEAD target TSSs.

Ontology analysis was performed as described previously (Gualdrini et al. 2016). Hypergeometric comparisons of target gene sets were performed in $\mathrm{R}$ using the phyper function. Numbers of overlapping genes were determined, and statistical significance was estimated with Bonferroni correction for multiple testing. Genes overexpressed in myCAFs (Ohlund et al. 2017) were defined as those commonly overexpressed greater than twofold in myCAFs compared with inflammatory CAFs and quiescent fibroblasts.

\section{Statistical analysis}

Analyses were performed using GraphPad Prism software. Mean values and standard error of the mean were generated from the indicated repeats of biological experiments. $P$-values were obtained from $t$-tests with unpaired samples, with a significance threshold of $P<0.05$.

\section{Acknowledgments}

We thank Fernando Calvo, Steve Hooper, and Erik Sahai for NFs and CAFs from the PyMT model, help with the contractility and invasion assays, and discussion; Probir Chakraborty for bioinformatic analysis; and Cyril Esnault, Caroline Hill, Erik Sahai, Nic Tapon, and Barry Thompson for discussions and comments on the manuscript. Work in the Treisman group was supported by Cancer Research UK until March 31, 2015, and since by the Francis Crick Institute (which receives its core funding from Cancer Research UK [FC001-190], the UK Medical Research Council [FC001-190], and the Wellcome Trust [FC001-190]) and ERC Advanced Grant 268690 ACTINonSRF to R.T.

\section{References}

Buxboim A, Swift J, Irianto J, Spinler KR, Dingal PC, Athirasala A, Kao YR, Cho S, Harada T, Shin JW, et al. 2014. Matrix elastic- ity regulates lamin-A,C phosphorylation and turnover with feedback to actomyosin. Curr Biol 24: 1909-1917.

Calvo F, Ege N, Grande-Garcia A, Hooper S, Jenkins RP, Chaudhry SI, Harrington K, Williamson P, Moeendarbary E, Charras G, et al. 2013. Mechanotransduction and YAP-dependent matrix remodelling is required for the generation and maintenance of cancer-associated fibroblasts. Nat Cell Biol 15: 637-646.

Calvo F, Ranftl R, Hooper S, Farrugia AJ, Moeendarbary E, Bruckbauer A, Batista F, Charras G, Sahai E. 2015. Cdc42EP3/ BORG2 and septin network enables mechano-transduction and the emergence of cancer-associated fibroblasts. Cell Rep 13: 2699-2714.

Cen B, Selvaraj A, Burgess RC, Hitzler JK, Ma Z, Morris SW, Prywes R. 2003. Megakaryoblastic leukemia 1, a potent transcriptional coactivator for serum response factor $(\mathrm{SRF})$, is required for serum induction of SRF target genes. Mol Cell Biol 23: 6597-6608.

Cordenonsi M, Zanconato F, Azzolin L, Forcato M, Rosato A, Frasson C, Inui M, Montagner M, Parenti AR, Poletti A, et al. 2011. The Hippo transducer TAZ confers cancer stem cell-related traits on breast cancer cells. Cell 147: 759-772.

Das A, Fischer RS, Pan D, Waterman CM. 2016. YAP nuclear localization in the absence of cell-cell contact is mediated by a filamentous actin-dependent, myosin II- and phospho-YAPindependent pathway during extracellular matrix mechanosensing. J Biol Chem 291: 6096-6110.

Discher DE, Mooney DJ, Zandstra PW. 2009. Growth factors, matrices, and forces combine and control stem cells. Science 324: 1673-1677.

Dong J, Feldmann G, Huang J, Wu S, Zhang N, Comerford SA, Gayyed MF, Anders RA, Maitra A, Pan D. 2007. Elucidation of a universal size-control mechanism in Drosophila and mammals. Cell 130: 1120-1133.

DuFort CC, Paszek MJ, Weaver VM. 2011. Balancing forces: architectural control of mechanotransduction. Nat Rev Mol Cell Biol 12: 308-319.

Dupont S. 2016. Role of YAP/TAZ in cell-matrix adhesion-mediated signalling and mechanotransduction. Exp Cell Res 343: 42-53.

Dupont S, Morsut L, Aragona M, Enzo E, Giulitti S, Cordenonsi M, Zanconato F, Le Digabel J, Forcato M, Bicciato S, et al. 2011. Role of YAP/TAZ in mechanotransduction. Nature 474: $179-183$.

Engler AJ, Sen S, Sweeney HL, Discher DE. 2006. Matrix elasticity directs stem cell lineage specification. Cell 126: 677-689.

Esnault C, Stewart A, Gualdrini F, East P, Horswell S, Matthews N, Treisman R. 2014. Rho-actin signaling to the MRTF coactivators dominates the immediate transcriptional response to serum in fibroblasts. Genes Dev 28: 943-958.

Farmer P, Bonnefoi H, Anderle P, Cameron D, Wirapati P, Becette V, Andre S, Piccart M, Campone M, Brain E, et al. 2009. A stroma-related gene signature predicts resistance to neoadjuvant chemotherapy in breast cancer. Nat Med 15: 68-74.

Ferrell JE Jr. 2008. Feedback regulation of opposing enzymes generates robust, all-or-none bistable responses. Curr Biol 18: R244-245.

Gaggioli C, Hooper S, Hidalgo-Carcedo C, Grosse R, Marshall JF, Harrington K, Sahai E. 2007. Fibroblast-led collective invasion of carcinoma cells with differing roles for RhoGTPases in leading and following cells. Nat Cell Biol 9: 1392-1400.

Gualdrini F, Esnault C, Horswell S, Stewart A, Matthews N, Treisman R. 2016. SRF co-factors control the balance between cell proliferation and contractility. Molecular cell 64: 1048-1061. 
Huang X, Yang N, Fiore VF, Barker TH, Sun Y, Morris SW, Ding Q, Thannickal VI, Zhou Y. 2012. Matrix stiffness-induced myofibroblast differentiation is mediated by intrinsic mechanotransduction. Am J Respir Cell Mol Biol 47: 340-348.

Jin F, Li Y, Dixon JR, Selvaraj S, Ye Z, Lee AY, Yen CA, Schmitt $\mathrm{AD}$, Espinoza CA, Ren B. 2013. A high-resolution map of the three-dimensional chromatin interactome in human cells. Nature 503: 290-294.

Johnson LA, Rodansky ES, Haak AJ, Larsen SD, Neubig RR, Higgins PD. 2014. Novel Rho/MRTF/SRF inhibitors block matrix-stiffness and TGF- $\beta$-induced fibrogenesis in human colonic myofibroblasts. Inflamm Bowel Dis 20: 154-165.

Judson RN, Tremblay AM, Knopp P, White RB, Urcia R, De Bari C, Zammit PS, Camargo FD, Wackerhage H. 2012. The Hippo pathway member Yap plays a key role in influencing fate decisions in muscle satellite cells. J Cell Sci 125: 6009-6019.

Kalluri R. 2016. The biology and function of fibroblasts in cancer. Nat Rev Cancer 16: 582-598.

Kaneko K, Ito M, Naoe Y, Lacy-Hulbert A, Ikeda K. 2014. Integrin av in the mechanical response of osteoblast lineage cells. Biochem Biophys Res Commun 447: 352-357.

Kim SY, Ferrell JE Jr. 2007. Substrate competition as a source of ultrasensitivity in the inactivation of Wee1. Cell 128: 1133-1145.

Kim NG, Gumbiner BM. 2015. Adhesion to fibronectin regulates Hippo signaling via the FAK-Src-PI3K pathway. I Cell Biol 210: $503-515$.

Kim T, Hwang D, Lee D, Kim JH, Kim SY, Lim DS. 2017. MRTF potentiates TEAD-YAP transcriptional activity causing metastasis. EMBO J 36: 520-535.

Latinkic BV, O'Brien TP, Lau LF. 1991. Promoter function and structure of the growth factor-inducible immediate early gene cyr61. Nucleic Acids Res 19: 3261-3267.

Levental KR, Yu H, Kass L, Lakins JN, Egeblad M, Erler JT, Fong SF, Csiszar K, Giaccia A, Weninger W, et al. 2009. Matrix crosslinking forces tumor progression by enhancing integrin signaling. Cell 139: 891-906.

Lim KP, Cirillo N, Hassona Y, Wei W, Thurlow JK, Cheong SC, Pitiyage G, Parkinson EK, Prime SS. 2011. Fibroblast gene expression profile reflects the stage of tumour progression in oral squamous cell carcinoma. J Pathol 223: 459-469.

Medjkane S, Perez-Sanchez C, Gaggioli C, Sahai E, Treisman R. 2009. Myocardin-related transcription factors and SRF are required for cytoskeletal dynamics and experimental metastasis. Nat Cell Biol 11: 257-268.

Meng Z, Moroishi T, Guan KL. 2016. Mechanisms of Hippo pathway regulation. Genes Dev 30: 1-17.

Miralles F, Posern G, Zaromytidou AI, Treisman R. 2003. Actin dynamics control SRF activity by regulation of its coactivator MAL. Cell 113: 329-342.

Moffitt RA, Marayati R, Flate EL, Volmar KE, Loeza SG, Hoadley KA, Rashid NU, Williams LA, Eaton SC, Chung AH, et al. 2015. Virtual microdissection identifies distinct tumor- and stroma-specific subtypes of pancreatic ductal adenocarcinoma. Nat Genet 47: 1168-1178.

O'Connor JW, Riley PN, Nalluri SM, Ashar PK, Gomez EW. 2015. Matrix rigidity mediates TGF $\beta 1$-induced epithelial-myofibroblast transition by controlling cytoskeletal organization and MRTF-A localization. J Cell Physiol 230: 1829-1839.

Ohlund D, Handly-Santana A, Biffi G, Elyada E, Almeida AS, Ponz-Sarvise M, Corbo V, Oni TE, Hearn SA, Lee EJ, et al. 2017. Distinct populations of inflammatory fibroblasts and myofibroblasts in pancreatic cancer. J Exp Med 214: 579-596.
Olson EN, Nordheim A. 2010. Linking actin dynamics and gene transcription to drive cellular motile functions. Nat Rev Mol Cell Biol 11: 353-365.

Ota M, Sasaki H. 2008. Mammalian Tead proteins regulate cell proliferation and contact inhibition as transcriptional mediators of Hippo signaling. Development 135: 4059-4069.

Panayiotou R, Miralles F, Pawlowski R, Diring J, Flynn H, Skehel M, Treisman R. 2016. Phosphorylation acts positively and negatively to regulate MRTF-A subcellular localisation and activity. Elife 5: e15460.

Paszek MJ, Zahir N, Johnson KR, Lakins JN, Rozenberg GI, Gefen A, Reinhart-King CA, Margulies SS, Dembo M, Boettiger D, et al. 2005. Tensional homeostasis and the malignant phenotype. Cancer Cell 8: 241-254.

Schedin P, Keely PJ. 2011. Mammary gland ECM remodeling, stiffness, and mechanosignaling in normal development and tumor progression. Cold Spring Harb Perspect Biol 3: a003228.

Schwartz MA. 2010. Integrins and extracellular matrix in mechanotransduction. Cold Spring Harb Perspect Biol 2: a005066.

Si Y, Ji X, Cao X, Dai X, Xu L, Zhao H, Guo X, Yan H, Zhang H, Zhu C, et al. 2017. Src inhibits the Hippo tumor suppressor pathway through tyrosine phosphorylation of Lats1. Cancer Res 77: 4868-4880.

Somogyi K, Rorth P. 2004. Evidence for tension-based regulation of Drosophila MAL and SRF during invasive cell migration. Dev Cell 7: 85-93.

Sotiropoulos A, Gineitis D, Copeland J, Treisman R. 1999. Signalregulated activation of serum response factor is mediated by changes in actin dynamics. Cell 98: 159-169.

Speight P, Kofler M, Szaszi K, Kapus A. 2016. Context-dependent switch in chemo/mechanotransduction via multilevel crosstalk among cytoskeleton-regulated MRTF and TAZ and TGF $\beta$-regulated Smad3. Nat Commun 7: 11642.

Stein C, Bardet AF, Roma G, Bergling S, Clay I, Ruchti A, Agarinis C, Schmelzle T, Bouwmeester T, Schubeler D, et al. 2015. YAP1 exerts its transcriptional control via TEAD-mediated activation of enhancers. PLOS Genet 11: e1005465.

Tominaga T, Sahai E, Chardin P, McCormick F, Courtneidge SA, Alberts AS. 2000. Diaphanous-related formins bridge Rho GTPase and Src tyrosine kinase signaling. Mol Cell 5: 13-25.

Vartiainen MK, Guettler S, Larijani B, Treisman R. 2007. Nuclear actin regulates dynamic subcellular localization and activity of the SRF cofactor MAL. Science 316: 1749-1752.

Wada K, Itoga K, Okano T, Yonemura S, Sasaki H. 2011. Hippo pathway regulation by cell morphology and stress fibers. $D e$ velopment 138: 3907-3914.

Wang Z, Wang DZ, Hockemeyer D, McAnally J, Nordheim A, Olson EN. 2004. Myocardin and ternary complex factors compete for SRF to control smooth muscle gene expression. $\mathrm{Na}$ ture 428: 185-189.

Yu FX, Zhao B, Panupinthu N, Jewell JL, Lian I, Wang LH, Zhao J, Yuan H, Tumaneng K, Li H, et al. 2012. Regulation of the Hippo-YAP pathway by G-protein-coupled receptor signaling. Cell 150: 780-791.

Yu OM, Miyamoto S, Brown JH. 2016. Myocardin-related transcription factor A and Yes-associated protein exert dual control in G protein-coupled receptor- and RhoA-mediated transcriptional regulation and cell proliferation. Mol Cell Biol 36: 39-49.

Zanconato F, Forcato M, Battilana G, Azzolin L, Quaranta E, Bodega B, Rosato A, Bicciato S, Cordenonsi M, Piccolo S. 2015. Genome-wide association between YAP/TAZ/TEAD and 
AP-1 at enhancers drives oncogenic growth. Nat Cell Biol 17: $1218-1227$.

Zaromytidou AI, Miralles F, Treisman R. 2006. MAL and ternary complex factor use different mechanisms to contact a common surface on the serum response factor DNA-binding domain. Mol Cell Biol 26: 4134-4148.

Zhang H, Liu CY, Zha ZY, Zhao B, Yao J, Zhao S, Xiong Y, Lei QY, Guan KL. 2009a. TEAD transcription factors mediate the function of TAZ in cell growth and epithelial-mesenchymal transition. J Biol Chem 284: 13355-13362.

Zhang X, Milton CC, Humbert PO, Harvey KF. 2009b. Transcriptional output of the Salvador/warts/hippo pathway is controlled in distinct fashions in Drosophila melanogaster and mammalian cell lines. Cancer Res 69: 6033-6041.
Zhang H, Pasolli HA, Fuchs E. 2011. Yes-associated protein (YAP) transcriptional coactivator functions in balancing growth and differentiation in skin. Proc Natl Acad Sci 108: 2270-2275.

Zhao XH, Laschinger C, Arora P, Szaszi K, Kapus A, McCulloch CA. 2007. Force activates smooth muscle $\alpha$-actin promoter activity through the Rho signaling pathway. I Cell Sci 120: 1801-1809.

Zhao B, Ye X, Yu J, Li L, Li W, Li S, Yu J, Lin JD, Wang CY, Chinnaiyan AM, et al. 2008. TEAD mediates YAP-dependent gene induction and growth control. Genes Dev 22: 1962-1971.

Zhao B, Li L, Wang L, Wang CY, Yu J, Guan KL. 2012. Cell detachment activates the Hippo pathway via cytoskeleton reorganization to induce anoikis. Genes Dev 26: 54-68. 


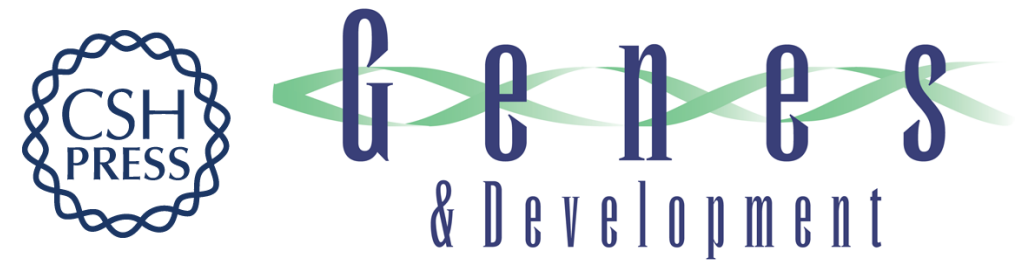

\section{Mutual dependence of the MRTF-SRF and YAP-TEAD pathways in cancer-associated fibroblasts is indirect and mediated by cytoskeletal dynamics}

Charles T. Foster, Francesco Gualdrini and Richard Treisman

Genes Dev. 2017, 31: originally published online January 9, 2018

Access the most recent version at doi:10.1101/gad.304501.117

Supplemental Material

References

Creative

Commons

License

Email Alerting

Service
http://genesdev.cshlp.org/content/suppl/2018/01/09/gad.304501.117.DC1

This article cites 60 articles, 22 of which can be accessed free at: http://genesdev.cshlp.org/content/31/23-24/2361.full.html\#ref-list-1

This article, published in Genes \& Development, is available under a Creative Commons License (Attribution 4.0 International), as described at http://creativecommons.org/licenses/by/4.0/.

Receive free email alerts when new articles cite this article - sign up in the box at the top right corner of the article or click here.

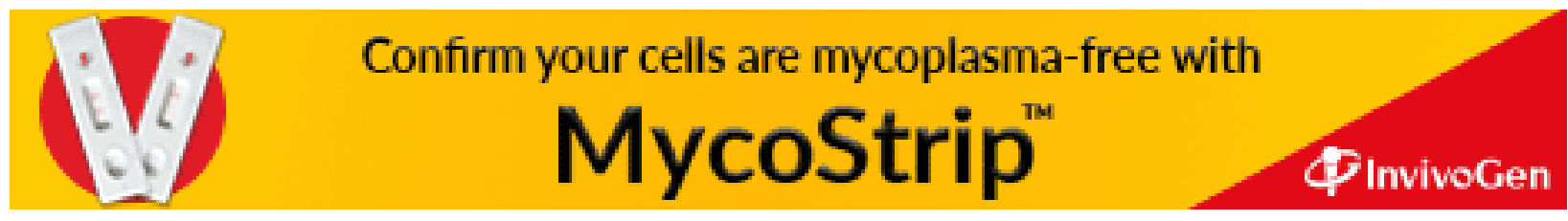

